\title{
The origin of the high metallicity of close-in giant exoplanets II The nature of the sweet spot for accretion
}

\author{
S. Shibata ${ }^{1,2}$, R. Helled ${ }^{1,}$, and M. Ikoma ${ }^{3,4,2}$ \\ 1 Institute for Computational Science (ICS), University of Zurich, e-mail: sho.shibata@uzh.ch \\ 2 Department of Earth and Planetary Science, Graduate School of Science, The University of Tokyo, 7-3-1 Hongo, Bunkyo-ku, Tokyo \\ 113-0033, Japan \\ 3 Division of Science, National Astronomical Observatory of Japan, 2-21-1 Osawa, Mitaka, Tokyo 181-8588, Japan \\ ${ }^{4}$ Department of Astronomical Science, The Graduate University for Advanced Studies (SOKENDAI), 2-21-1 Osawa, Mitaka, Tokyo \\ 181-8588, Japan
}

Received XXX XX, 2021; accepted XXXXX XX, 2021

\section{ABSTRACT}

\begin{abstract}
Context. The composition of gas giant planets reflects their formation and evolution history. Revealing the origin of the high heavyelement masses in giant exoplanets is a objective of planet formation theories. Planetesimal accretion during the phase of planetary migration could lead to the delivery of heavy elements into gas giant planets. In our previous paper (Shibata et al. 2020) we used dynamical simulations and showed that planetesimal accretion during planetary migration occurs in a rather narrow region of the protoplanetary disk, which we refer as "the sweet spot for accretion".

Aims. Our understanding of the sweet spot, however, is still limited. The location of the sweet spot within the disk and how it changes as the disk evolves was not investigated in detail. The goal of this paper is to reveal the nature of the sweet spot using analytical calculations and investigate the role of the sweet spot in determining the composition of gas giant planets.

Methods. We analytically derive the required conditions for the sweet spot. Then, using the numerical integration of the orbits of planetesimals around a migrating planet, we compare the derived equations with the numerical results.

Results. We find that the conditions required for the sweet spot can be expressed by the ratio of the aerodynamic gas damping timescale of the planetesimal orbits and the planetary migration timescale. If the planetary migration timescale depends on the surface density of disk gas inversely, the location of the sweet spot does not change with the disk evolution. We expect that the planets observed inner to the sweet spot include much more heavy elements than the planets outer to that. The mass of planetesimals accreted by the protoplanet in the sweet spot depends on the amount of planetesimals that are shepherded by mean motion resonances. Our analysis suggests that tens Earth-mass of planetesimals can be shepherded into the sweet spot without planetesimal collisions. However, as more planetesimals are trapped into mean motion resonances, collisional cascade can lead to fragmentation and the production of smaller planetesimals. This could affect the location of the sweet spot and the population of small objects in planetary systems. Conclusions. We conclude that the composition of gas giant planets depends on whether the planets crossed the sweet spot during their formation. Constraining the metallicity of cold giant planets, that are expected to be outer than the sweet spot, with future observations would reveal key information for understanding the origin of heavy elements in giant planets.
\end{abstract}

Key words. giant planet formation - planetary migration

\section{Introduction}

The formation of gas giant planets is a complex process which involves many underlying physical processes, such as core formation, gas accretion, and planetary migration. Recent planetary population synthesis models (e.g., Ida et al. 2018, Mordasini 2018) aim to consider as many processes as possible and construct a comprehensive planetary formation model which begins at the early stages of planetesimal accretion up to the formation of gas giant planets. However, there are still many uncertainties related to each of these processes as well as the initial conditions. Constraining theories using observed physical parameters is crucial for understanding the formation and evolution of gas giant planets. Along with the development of observation and characterisation of exoplanets, many studies aimed to validate theoretically proposed formation models and constrain the initial parameters that control giant planet formation. The planetary chemical composition is a key characteristic that can be used to constrain formation models because the formation history is imprinted in the composition of gas giant planets.

Recent observations provide information on the chemical composition of gas giant planets. Typically it is the planetary metallicity is used for their characterisation and for constraining their formation and evolution histories (e.g., Helled et al. 2021). The composition of gas giant planets was first constrained for the solar-system giant planets Jupiter and Saturn (e.g. Guillot 1999, Saumon \& Guillot 2004). From the gravitational moments observed by spacecrafts, the total heavy-element mass in Jupiter $M_{\mathrm{Z}, \mathrm{Jup}}$ was estimated to be $\sim 25-45 M_{\oplus}$ (Wahl et al. 2017, Debras \& Chabrier 2019) and $M_{\mathrm{Z}, \mathrm{Sat}} \sim 16-30 M_{\oplus}$ for Saturn (Saumon \& Guillot 2004, Helled \& Guillot 2013; Movshovitz et al. 2020; Mankovich \& Fuller 2021). Heavy-element enrichment has also been inferred for giant exoplanets (e.g., Guillot et al. 2006; Miller \& Fortney 2011, Thorngren et al. 2016). According to Thorngren et al. (2016), close-in gas giant planets contain large amounts of heavy elements of the order of tens 
of Earth masses, with bulk planetary metallicities that are significantly higher than stellar metallicities. Surprisingly, some of these planets are estimated to consist of more than $\sim 100 M_{\oplus}$ of heavy elements. Revealing the origin of this significant heavyelement enrichment in giant planets is a key objective in giant planet formation theory.

Planetesimal accretion has been proposed to be one of the main sources of heavy-element enrichment of giant planets. After the onset of runaway gas accretion, a large amount of planetesimals enters the expanding feeding zone where the planetesimals can be captured by the protoplanet (Zhou \& Lin 2007; Shiraishi \& Ida 2008, Shibata \& Ikoma 2019, Podolak et al. 2020). During the rapid gas accretion phase, up to $30 \%$ of planetesimals inside the feeding zone are captured by the protoplanet (Shibata \& Ikoma 2019). For a massive protoplanetary disk, this accretion process is sufficient to explain the estimated heavy-element masses in Jupiter and Saturn. For close-in giant exoplanets, however, the heavy-element mass that accreted during rapid gas accretion is rather low because the total mass planetesimals inside the feeding zone of these planets is significantly lower.

During the planetary migration phase, migrating planets encounter planetesimals in wide regions within the protoplanetary disk. Planetesimal accretion during the migration phase was investigated in the context of accelerating the formation of Jupiter's core (e.g. Tanaka \& Ida 1999; ; Alibert et al. 2005). It was found that the formation timescale of Jupiter's core can be shortened if planetary migration is included. For the origin of large amounts of heavy elements in close-in giant exoplanets, planetesimal accretion by a migrating Jupiter-size planet was also investigated by Shibata et al. (2020) and Turrini et al. (2021). Shibata et al. (2020) performed orbital integration calculations of planetesimals around a migrating planet, and found that several-tens Earth mass of planetesimals can be accreted by a gas giant planet if the planet migrates tens of $\mathrm{AU}$ in a massive protoplanetary disk. Turrini et al. (2021) also investigated this process and found that planetesimal accretion during planetary migration is a useful tracer of the formation location of giant planets. Thus, planetesimal accretion during the planetary migration phase is a key mechanism in the formation history of gas giant planets.

If the migrating planet is as massive as Jupiter, efficient planetesimal accretion is limited to a narrow ring-like region within the protoplanetary disk (Shibata et al. 2020); in this paper, we refer to this region as the sweet spot for planetesimal accretion (hereafter, SSP). Even in the case of a migrating Earthmass planet, the accretion area is limited to an outer disk region (without outer edge) because of the disk gas drag (Tanaka \& Ida 1999). However, such a ring-like region of SSP is specific to the Jupiter-mass planet. The shape and location of the accretion region during the planetary migration determine the accretion area and the accretion efficiency. The accretion area of planets with different masses depends on the strength of mean motion resonances accompanied by the migrating planet. Nevertheless, the detailed nature of SSP is not fully understood.

In this paper, we investigate the nature of SSP in detail and derive analytical equations for determining its location. In sec.2, we analytically investigate the orbital evolution of planetesimals around the migrating planet. We derive a condition required for planetesimal accretion during the planetary migration phase. In sec. 3. we compare the derived condition with the numerical simulations. In sec. 4, we discuss about the location of SSP in a protoplanetary disk and the heavy-element enrichment of giant planets. Our conclusions are summarised in Section 5.

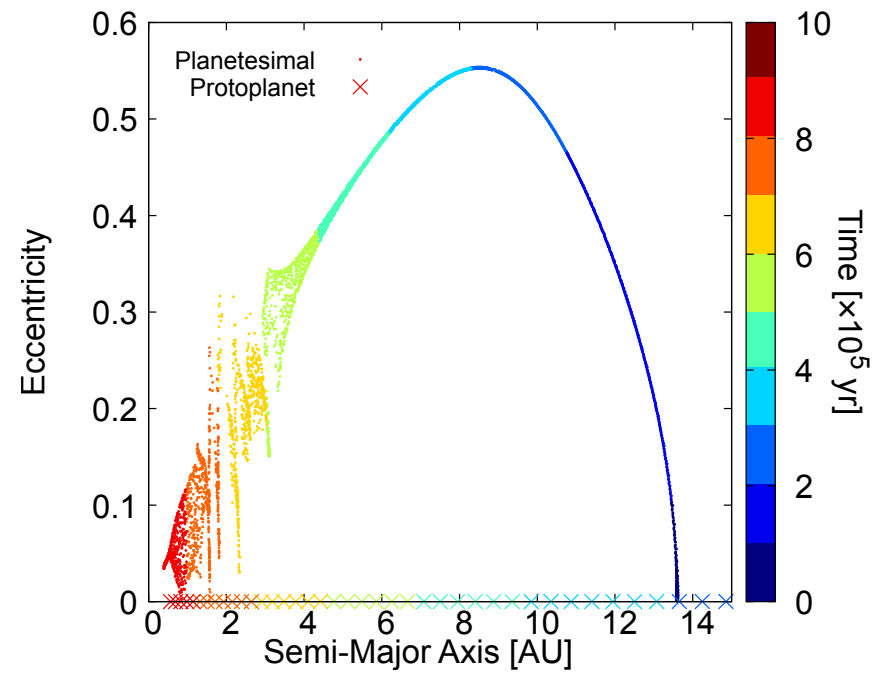

Fig. 1. Orbital evolution of a planetesimal initially located at $13.8 \mathrm{AU}$ dictated via the gravitational interaction with a migrating Jupiter-mass protoplanet. The dots and crosses show the locations of the planetesimal and the protoplanet, respectively, and are colour-coded according to time. In this simulation the surface density of disk gas is set to that of the minimum-mass solar nebula and aerodynamic gas drag is included. Here the protoplanet migrates from 15.0AU with migration rate of $10^{5}$ $\mathrm{AU} / \mathrm{yr}$. For the details of the numerical simulation, see sec. 3

\section{An analytical expression of the accretion sweet spot}

\subsection{An overview of planetesimal accretion during planetary migration}

Mean motion resonances play an important role in the accretion of planetesimals onto a migrating protoplanet. Here, we summarise the orbital evolution of planetesimals dictated via their gravitational interaction with a migrating protoplanet, which as found by the numerical simulations of Shibata et al. (2020). Figure 1 shows the orbital evolution of a planetesimal initially located at $13.8 \mathrm{AU}$ around a migrating Jupiter-mass protoplanet. The orbital evolution of the planetesimal is divided into three characteristic phases as discussed below.

First phase $\left(t=0 \mathrm{yr}-4 \times 10^{5} \mathrm{yr}\right.$; bluish symbols $)$ : The planetesimal encounters the migrating protoplanet and is trapped in the $2: 1$ mean motion resonance; this phenomenon is referred to as the resonant trapping. The planetesimal trapped in the mean motion resonance is transported inward together with the migrating protoplanet and its eccentricity is highly enhanced. This phenomenon is known as the resonant shepherding (Batygin \& Laughlin 2015).

Second phase $\left(t=4 \times 10^{5} \mathrm{yr}-7 \times 10^{5} \mathrm{yr}\right.$; green-yellow symbols): As time progresses the resonantly-trapped planetesimal starts to escape from the mean motion resonance, and its eccentricity begins to decrease. This is because of the stronger aerodynamic drag in the inner regions. The break of resonant trapping shown in Fig. 11 is caused by overstable liblation (Goldreich \& Schlichting 2014). The eccentricity of the trapped planetesimal is excited through gravitational scattering by the migrating protoplanet but is also damped by the disk gas drag. This overstable equilibrium condition makes the planetesimal orbit unstable and breaks the resonant trapping. 
Third phase $\left(t=7 \times 10^{5} \mathrm{yr}-;\right.$ reddish symbols): In the farther inner region, the disk gas is dense enough that the resonantlytrapped planetesimal is damped faster than the planetary migration and, therefore, the planetesimal is eliminated from the feeding zone. We refer to this phenomenon as the aerodynamic shepherding, which was found by Tanaka \& Ida(1999) in the context of terrestrial planet formation.

According to the numerical simulations preformed in Shibata et al. (2020), planetesimal accretion occurs during the second phase, and the region in which for efficient planetesimal accretion occurs is referred to as SSP. During the first and third phases, planetesimal accretion is negligible. The existence of these three different phases (or regions) is associated with the change in strength of the aerodynamic gas drag which increases as the protoplanet migrates inward.

In the following sections, we derive the conditions for the SSP analytically. In Sec. 2.2 we show that planetesimals are generally trapped in the mean motion resonances with an approaching protoplanet in standard protoplanetary disks. In Sec. 2.3. we show that the eccentricity of the resonantly-trapped planetesimal reaches an equilibrium value, $e_{\mathrm{eq}}$, that is determined by the ratio of the aerodynamic damping timescale (or the friction timescale) for the planetesimal $\left(\tau_{\text {aero, }, 0}\right.$; Eq. [16]) and the tidal damping timescale (or the migration timescale) of the protoplanet $\left(\tau_{\text {tide, }, a}\right)$. A necessary condition for a planetesimal to be captured is that it enters the feeding zone of the protoplanet. As for the resonantly trapped planetesimal, $e_{\mathrm{eq}}$ must exceed the eccentricity at the feeding zone boundary $\left(e_{\text {cross }}\right.$; Eq. [27]), which is derived in Sec. 2.4. Even if such a condition is satisfied, however, for the trapped planetesimal to escape the resonance completely, the perturbed planetesimal's orbit must oscillate more widely relative to the resonant width (i.e., the overstable libration) and such an oscillation must be amplified more rapidly than the protoplanet's migration. The conditions are derived in Sec. 2.5. Finally, by putting everything above together, we infer the condition for the SSP in Sec. 2.6.

\subsection{Before resonant trapping}

When the radial distance between two objects decreases, which is referred to as convergent orbital evolution, the objects are eventually locked in a resonant state. Such resonant trapping has been discussed in the contexts of the formation of satellite pairs (e.g., Goldreich 1965; Dermott et al. 1988; Malhotra 1993b), the transport of small bodies (e.g., Yu \& Tremaine 2001;|Batygin \& Laughlin 2015), and the formation of exoplanet pairs (e.g., Fabrycky et al. 2014; Goldreich \& Schlichting 2014, Batygin|2015, and references therein). However, not all resonant encounters result in resonant trapping. The resonant trapping requires the following conditions for a planetesimal-protoplanet pair (e.g. Malhotra 1993a):

(i) The two orbits converge with each other;

(ii) The eccentricity of the planetesimal, $e$, before it is trapped in the resonance is smaller than the critical value $e_{\text {crit }}$ (see Eq. (2));

(iii) The timescale for the planetesimal to cross the resonant width $\tau_{\text {cross }}$ is longer than the libration timescale $\tau_{\text {lib }}$ (see Eqs. (3) and (8)).

Here, we consider a trap of a planetesimal in the inner first-order mean motion resonances $(j: j-1$ resonance) with a protoplanet migrating inward and assume the mass of the planetesimal is negligibly small relative to that of the protoplanet.
The first condition can be obtained by comparing the timescales of change in the semi-major axis of the planetesimal and the protoplanet. In a protoplanetary gaseous disk, the orbits of objects shrink by the aerodynamic gas drag and gravitational tidal drag from the disk gas. The former drag is dominant for planetesimal-size objects $\left(\lesssim 10^{24} \mathrm{~g}\right)$ and the latter drag is for planet-size objects $\left(\gtrsim 10^{24} \mathrm{~g}\right)($ Zhou \& Lin 2007). Using the aerodynamic damping timescale for semi-major axis $\tau_{\text {aero }, a}$ for the planetesimal and the tidal damping timescale for semi-major axis $\tau_{\text {tide }, a}$ for the protoplanet, we can write the first condition as:

$\tau_{\text {aero }, a}>\tau_{\text {tide }, a}$.

As for the second condition, the critical eccentricity for the $j: j-1$ resonances is given by (e.g. Murray \& Dermott 1999):

$e_{\text {crit }}=\sqrt{6}\left[\frac{3}{\left|f_{\mathrm{d}}\right|}(j-1)^{4 / 3} j^{2 / 3} \frac{M_{\mathrm{s}}}{M_{\mathrm{p}}}\right]^{-1 / 3}$,

where $M_{\mathrm{p}}$ and $M_{\mathrm{s}}$ are the masses of the planet and central star, respectively, and $f_{\mathrm{d}}$ is the interaction coefficient whose values are summarised in Table 1

When $\tau_{\text {aero, } a} \gg \tau_{\text {tide, } a}$, the timescale for the planetesimal to cross the resonant width $\tau_{\text {cross }}$ is given by:

$\tau_{\text {cross }}=\left|\frac{\Delta a_{\text {res }}}{\dot{a}_{\mathrm{c}}}\right|=\left|\frac{\Delta a_{\text {res }}}{a_{\mathrm{c}}}\right| \tau_{\text {tide }, a}$,

where $a_{\mathrm{c}}$ is the semi-major axis of the resonance centre, which is related to the protoplanet's semi-major axis $a_{\mathrm{p}}$ as:

$a_{\mathrm{c}}=\left(\frac{j-1}{j}\right)^{2 / 3} a_{\mathrm{p}}$

$\dot{a}_{\mathrm{c}}$ is the temporal change of $a_{\mathrm{c}}$, and $\Delta a_{\mathrm{res}}$ is the width of the resonance given by (e.g. Murray \& Dermott 1999):

$$
\begin{aligned}
\frac{\Delta a_{\mathrm{res}}}{a_{\mathrm{c}}}=2 & \left\{\frac{16}{3} \frac{M_{\mathrm{p}}}{M_{\mathrm{s}}}\left(\frac{j-1}{j}\right)^{2 / 3} e\left|f_{\mathrm{d}}\right|\right\}^{1 / 2} \\
& \left\{1+\frac{1}{27(j-1)^{2} e^{3}} \frac{M_{\mathrm{p}}}{M_{\mathrm{s}}}\left(\frac{j-1}{j}\right)^{2 / 3}\left|f_{\mathrm{d}}\right|\right\}^{1 / 2} .
\end{aligned}
$$

The width of the resonance depends on the eccentricity and takes the minimum value at:

$e=\frac{2^{1 / 3}}{3}\left(|j|^{-2 / 3}|j-1|^{-4 / 3}\left|f_{\mathrm{d}}\right| \frac{M_{\mathrm{p}}}{M_{\mathrm{s}}}\right)^{1 / 3}$.

By substituting Eq. (6) into Eq. (5), we obtain the minimum width of the resonance as:

$$
\left.\frac{\Delta a_{\mathrm{res}}}{a_{\mathrm{c}}}\right|_{\min }=\frac{2^{8 / 3}}{3^{1 / 2}} j^{-4 / 9}(j-1)^{1 / 9}\left|f_{\mathrm{d}}\right|^{2 / 3}\left(\frac{M_{\mathrm{p}}}{M_{\mathrm{s}}}\right)^{2 / 3} \text {. }
$$

The libration timescale at internal resonances are given by (e.g. Murray \& Dermott 1999; Batygin \& Laughlin 2015):

$$
\begin{aligned}
\tau_{\text {lib }}=\frac{2 \pi}{n} & \mid\left(\frac{j-1}{j}\right)^{4 / 3}\left(\frac{M_{\mathrm{p}}}{M_{\mathrm{s}}}\right)^{2} \frac{f_{\mathrm{d}}^{2}}{e^{2}} \\
& -\left.3(j-1)^{2}\left(\frac{j-1}{j}\right)^{2 / 3} \frac{M_{\mathrm{p}}}{M_{\mathrm{s}}} f_{\mathrm{d}} e\right|^{-1 / 2},
\end{aligned}
$$




\begin{tabular}{c|c|c}
\hline$p+q: p$ & $f_{\mathrm{s}}$ & $f_{\mathrm{d}}$ \\
\hline $2: 1$ & 0.387627 & -1.19049 \\
$3: 2$ & 1.15279 & -2.02521 \\
$4: 3$ & 2.27923 & -2.84042 \\
$5: 4$ & 3.76541 & -3.64962 \\
\hline
\end{tabular}

Table 1. Values of the secular term $f_{\mathrm{s}}$ and the direct term $f_{\mathrm{d}}$ for the first order mean motion resonances.

where $n$ is the planetesimals' mean motion. Substituting Eq. (6) into Eq. (8), we obtain the corresponding libration timescale as:

$\tau_{\text {lib }}=0.48 j^{4 / 9}(j-1)^{-10 / 9}\left(\frac{M_{\mathrm{p}}}{M_{\mathrm{s}}}\right)^{-2 / 3}\left|f_{\mathrm{d}}\right|^{-2 / 3} T_{\mathrm{K}}$.

where $T_{\mathrm{K}}(=2 \pi / n)$ is the Kepler period at the resonance centre. Using Eqs. (3), (7) and (9), we obtain the third (sufficient) condition as

$\frac{\tau_{\text {cross }}}{\tau_{\text {lib }}}=7.7 j^{1 / 9}(j-1)^{2 / 9}\left|f_{\mathrm{d}}\right|^{4 / 3}\left(\frac{M_{\mathrm{p}}}{M_{\mathrm{s}}}\right)^{4 / 3} \frac{\tau_{\text {tide }, a}}{T_{\mathrm{K}, \mathrm{p}}} \gtrsim 1$,

where $T_{\mathrm{K}, \mathrm{p}}$ is the Kepler period of the migrating protoplanet; namely, $T_{\mathrm{K}, \mathrm{p}}=T_{\mathrm{K}} j /(j-1)$.

Far from the protoplanet and outside mean motion resonances, the planetesimal's eccentricity is determined by the balance between the viscous stirring from the planetesimal swarm and the aerodynamic gas drag. The mean value of r.m.s. eccentricities of the planetesimal swarm is on the order of $\lesssim 10^{-2}$ (Ohtsuki et al.2002). For km-sized planetesimals with $e=10^{-2}$, the radial drift timescale $\tau_{\mathrm{damp}, a}$ is longer than the planetary migration timescale in the type II regime $\left(\tau_{\text {tide }, a} \lesssim 10^{7} \mathrm{yr}\right)$ over a wide region of a protoplanetary disk (Adachi et al. 1976). Also, the critical eccentricity is $e_{\text {crit }} \sim 0.15$ for $j=2$ and $M_{\mathrm{p}} / M_{\mathrm{s}}=10^{-3}$. Thus, the first and second conditions are expected to be achieved in protoplanetary disks. Figure 2 shows the timescale ratio $\tau_{\text {cross }} / \tau_{\text {lib }}$ as a function of semi-major axis, calculated from Eq. (10). Here, we show the cases of $2: 1$ (thick lines) and $3: 2$ (thin lines) resonances, and consider the type II regime for the migration timescale $\tau_{\text {tide }, a}$ obtained in Kanagawa et al. (2018). To calculate $\tau_{\text {tide, } a}$, we adopt the self-similar solution of Lynden-Bell \& Pringle (1974) for the disk structure with a disk accretion rate of $10^{-8} M_{\odot} / \mathrm{yr}$ and viscosity of $10^{-3}$. The disk aspect ratio is set to $0.03 r^{1 / 4}$. The timescales ratio $\tau_{\text {cross }} / \tau_{\text {lib }}$ is larger than unity except for the cases of $M_{\mathrm{p}} / M_{\mathrm{s}}=10^{-4}$ in outer disk $a \gg 10 \mathrm{AU}$. Thus, we conclude that the resonant trapping of planetesimals usually occurs during the migration of a giant protoplanet.

\subsection{Orbital evolution under resonant trapping}

Trapped planetesimals suffer from gravitational perturbation from the migrating protoplanet and aerodynamic gas drag from the disk gas. The Lagrange's equations under the aerodynamic gas drag are given by (e.g., Murray \& Dermott 1999):

$\dot{a}=2(j-1) C_{\mathrm{r}} a e \sin \varphi-\frac{a}{\tau_{\mathrm{aero}, a}}$,

$\dot{e}=-C_{\mathrm{r}} \sin \varphi-\frac{e}{\tau_{\text {aero,e }}}$,

where $\varphi$ is the resonant angle and

$C_{\mathrm{r}}=\frac{M_{\mathrm{p}}}{M_{\mathrm{s}}} n \alpha f_{\mathrm{d}}$

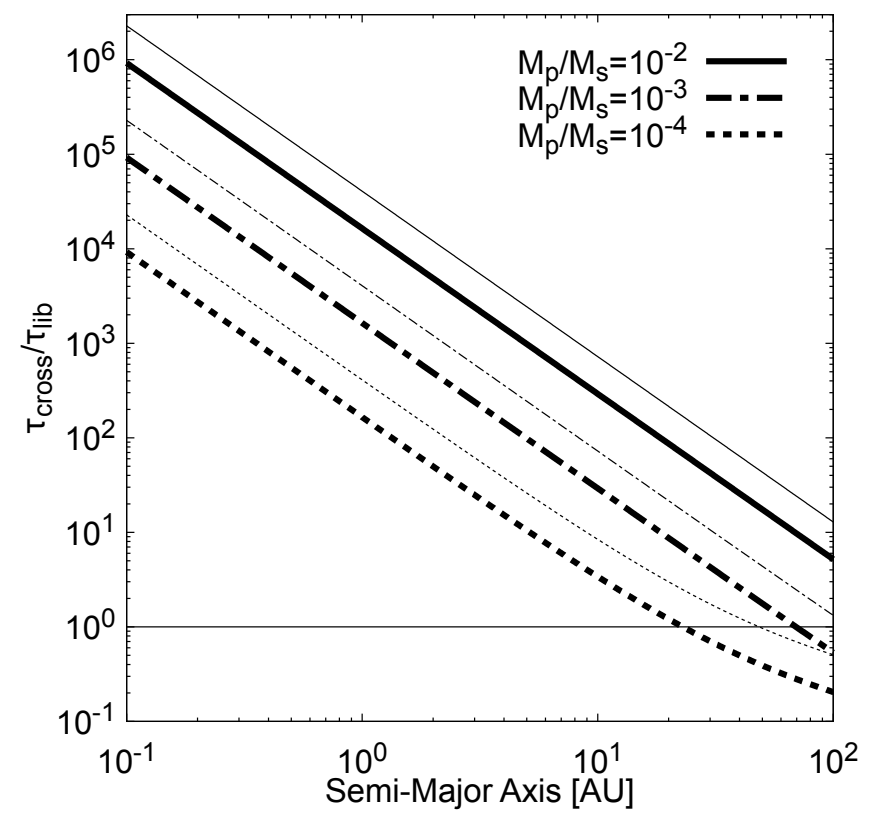

Fig. 2. The ratio between the crossing timescale $\tau_{\text {cross }}$ (Eq. 3 and the libration timescale $\tau_{\text {lib }}$ (Eq. 9) as a function of the planetesimal's semimajor axis. Here, we consider the type II regime obtained in Kanagawa et al. (2018) for the migration timescale $\tau_{\text {tide, } a}$. Solid, dash-dotted, and dotted lines show the cases of the planet-to-star mass ratio $M_{\mathrm{p}} / M_{\mathrm{s}}=$ $10^{-2}, 10^{-3}$ and $10^{-4}$, respectively. We consider the inner first-order mean motion resonances (or $j: j-1$ resonances); thick lines are for $j=2$ and thin lines are for $j=3$. To calculate the migration timescale, we used a disk model same as that in Ida et al. (2018). The disk accretion rate, disk's viscosity parameter, and disk aspect ratio are set to $10^{-8} M_{\odot} / \mathrm{yr}$, $10^{-3}$ and $0.03 r^{1 / 4}$, respectively.

$\tau_{\text {aero }, a}$ and $\tau_{\text {aero, } e}$ are the aerodynamic damping timescales for semi-major axis and eccentricity, which are given by (Adachi et al. 1976):

$\tau_{\text {aero }, a}=\frac{\tau_{\text {aero }, 0}}{2}\left\{\left(0.97 e+0.64 i+\left|\eta_{\text {gas }}\right|\right) \eta_{\text {gas }}\right\}^{-1}$,
$\tau_{\text {aero }, e}=\tau_{\text {aero }, 0}\left(0.77 e+0.64 i+1.5\left|\eta_{\text {gas }}\right|\right)^{-1}$,

where

$\tau_{\text {aero }, 0}=\frac{2 m_{\mathrm{pl}}}{C_{\mathrm{d}} \pi R_{\mathrm{pl}}^{2} \rho_{\mathrm{gas}} \nu_{\mathrm{K}}}$.

Here $C_{\mathrm{d}}$ is the non-dimensional drag coefficient, $m_{\mathrm{pl}}$ is the planetesimal's mass, $R_{\mathrm{pl}}$ is the planetesimal's radius, $\rho_{\mathrm{gas}}$ is the gas density, and $v_{\mathrm{K}}$ is the Kepler velocity. The planetesimal's mass $m_{\mathrm{pl}}$ is calculated as $4 \pi \rho_{\mathrm{pl}} R_{\mathrm{pl}}{ }^{3} / 3$, where $\rho_{\mathrm{pl}}$ is the planetesimal's mean density. $\eta_{\text {gas }}$ is a parameter that defines the sub-Keplerian velocity of disk gas as:

$v_{\text {gas }}=v_{\mathrm{K}}\left(1-\eta_{\mathrm{gas}}\right)$.

Note that we have neglected the high-order terms in eccentricity in Eqs. (11), (12), (14) and (15). Although the eccentricities of the trapped planetesimals are easily excited to $e^{2} \sim 0.1$, the location of the sweet spot analytically derived with these equations is found to be consistent with the numerical results, as shown in section 3 . 
During the resonant trapping, the period ratio between the protoplanet and trapped planetesimal remains constant, by definition, and

$\frac{a}{\dot{a}}=\frac{a_{\mathrm{p}}}{\dot{a}_{\mathrm{p}}}=-\tau_{\text {tide, } a}$.

When $\tau_{\text {aero }, a} \gg \tau_{\text {tide, } a}$, using Eq. (18), we can re-write Eq. (11) as:

$\sin \varphi=-\frac{1}{2(j-1) C_{\mathrm{r}} e \tau_{\mathrm{tide}, a}}$.

Also, using Eq. (19) in Eq. [12, we obtain:

$\dot{e}=\frac{1}{2(j-1) e \tau_{\text {tide }, a}}-\frac{e}{\tau_{\text {aero }, e}}$.

Just after trapped into the resonances, $e$ is small and $\dot{e}$ is positive. Equation 20 indicates that $e$ increases on a timescale $\sim e^{2} \tau_{\text {tide }, a}\left(\ll \tau_{\text {tide }, a}\right)$ and reaches an equilibrium value. Solving Eqs. (19) and (20) with $\dot{e}=0$, we obtain the equilibrium eccentricity and resonant argument given by:

$$
\begin{aligned}
e_{\mathrm{eq}} & =\left\{\frac{1}{2(j-1)} \frac{\tau_{\mathrm{aero}, e}}{\tau_{\text {tide }, a}}\right\}^{1 / 2}, \\
\sin \varphi_{\mathrm{eq}} & =-\frac{e_{\mathrm{eq}}}{C_{\mathrm{r}} \tau_{\mathrm{aero}, e}} .
\end{aligned}
$$

Unlike the eccentricity, the planetesimals' inclinations are not excited by the migrating protoplanet because the planetesimals are far from the protoplanet. When $e \gg i$ and $e \gg \eta_{\mathrm{gas}}$, $\tau_{\text {aero }, e}=\tau_{\text {aero, } 0}(0.77 e)^{-1}$ from Eq. (15) and, thus, $e_{\mathrm{eq}}$ and $\sin \varphi_{\mathrm{eq}}$ are written approximately as:

$$
\begin{aligned}
e_{\mathrm{eq}} & \sim\left\{\frac{1}{1.54(j-1)} \frac{\tau_{\mathrm{aero}, 0}}{\tau_{\text {tide }, a}}\right\}^{1 / 3}, \\
\sin \varphi_{\mathrm{eq}} & \sim-\frac{0.77 e_{\mathrm{eq}}^{2}}{C_{\mathrm{r}} \tau_{\mathrm{aero}, 0}} .
\end{aligned}
$$

Note that if the high-order terms of the eccentricity are considered, the equilibrium eccentricity is smaller than that given by Eq. 23.

\subsection{Aerodynamic shepherding}

As shown in Shibata et al. (2020), the mean motion resonances play a role like narrow flow channels for planetesimals to enter the planet's feeding zone; the channels are called the accretion bands. Mathematically speaking, planetesimals with negative values of the Jacobi energy, $E_{\text {Jacobi }}$, can enter the feeding zone, once their eccentricities are sufficiently enhanced so that their Jacobi energy becomes positive. The Jacobi energy is given by (Hayashi et al. 1977):

$E_{\mathrm{Jacobi}}=\frac{\mathcal{G} M_{\mathrm{s}}}{a_{\mathrm{p}}}\left\{-\frac{a_{\mathrm{p}}}{2 a}-\sqrt{\frac{a}{a_{\mathrm{p}}}\left(1-e^{2}\right)} \cos i+\frac{3}{2}+\frac{9}{2} h^{2}+O\left(h^{3}\right)\right\}$,

where $h$ is the reduced Hill radius defined as

$h=\left(\frac{M_{\mathrm{p}}}{3 M_{\mathrm{s}}}\right)^{1 / 3}$.

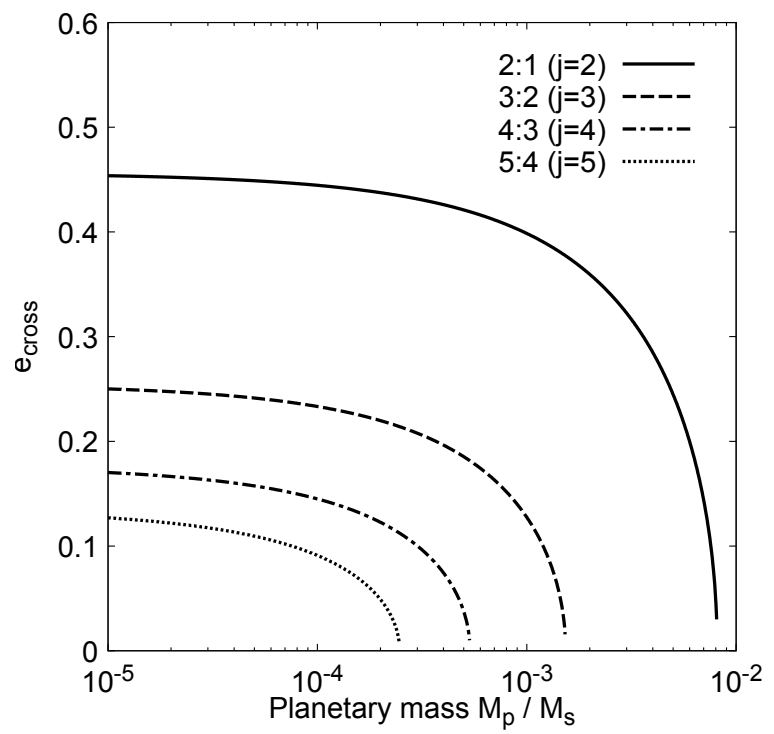

Fig. 3. The dependence of the crossing point eccentricity $e_{\text {cross }}$ (see Eq. 27 on the planet-to-star mass ratio $M_{\mathrm{p}} / M_{\mathrm{s}}$ for different four cases of $j=2$ (solid), $j=3$ (dashed), $j=4$ (dash-dotted) and $j=5$ (dotted), respectively.

Substituting $E_{\text {jacobi }}=0$ and $a / a_{\mathrm{p}}=\{(j-1) / j\}^{2 / 3}$ into Eq. 25 and assuming $\cos ^{2} i \sim 1$, one obtains the eccentricity of the accretion band, $e_{\text {cross }}$, as:

$e_{\text {cross }}=\left[1-\left(\frac{j}{j-1}\right)^{2 / 3}\left\{\frac{3}{2}+\frac{9}{2} h^{2}-\frac{1}{2}\left(\frac{j}{j-1}\right)^{2 / 3}\right\}^{2}\right]^{1 / 2}$.

The condition required for entering the feeding zone is that the equilibrium eccentricity $e_{\mathrm{eq}}$ (see Eq. 23) is larger than $e_{\mathrm{cross}}$, which is given by:

$1.54(j-1) e_{\text {cross }}^{3}<\frac{\tau_{\text {aero }, 0}}{\tau_{\text {tide }, a}}$.

Figure 3 shows $e_{\text {cross }}$ as a function of $M_{\mathrm{p}} / M_{\mathrm{s}}$ for different values of $j$ from Eq. 27). As indicated in this figure, $e_{\text {cross }}$ decreases with increasing planetary mass. This is because as the protoplanet mass increases, the feeding zone expands and, consequently, the crossing point (i.e., the accretion band) itself is located inside the feeding zone, once the planetary mass exceeds a certain value. For example, while there are three crossing points ( $j=2,3,4)$ when $M_{\mathrm{p}} / M_{\mathrm{s}}=10^{-3.5}$, there is only one crossing point $(j=2)$ when $M_{\mathrm{p}} / M_{\mathrm{s}}=10^{-2.5}$ and no points when $M_{\mathrm{p}} / M_{\mathrm{s}}=10^{-2}$.

\subsection{Resonant breaking}

Even when entering the feeding zone, planetesimals trapped in the mean motion resonances are not always accreted by the migrating protoplanet. The conjunction point of a trapped planetesimal with the protoplanet is given in terms of the mean longitude as:

$\lambda_{\text {con }}=\varphi+\varpi$,

where $\varpi$ is the longitude of pericenter. The resonant argument, $\varphi$, which is the displacement of the longitude of conjunction 
from the pericenter, takes $\varphi_{\mathrm{eq}} \sim 0$ during the converging orbital evolution. This means that every conjunction occurs at the pericenter of the planetesimal orbit; namely, the planetesimal's orbit is far from the protoplanet's one at the conjunction. Thus, trapped planetesimals cannot enter the Hill sphere of the protoplanet and planetesimal accretion does not occur (see Figs. C.1 and C.2 in Shibata et al. 2020).

A planetesimal is captured by the migrating protoplanet only if the resonant trapping is broken. Under the forces damping and enhancing the planetesimal's eccentricity, the orbit of the trapped planetesimal becomes unstable and starts to oscillate. Once the amplitude of oscillation exceeds the resonant width, the resonant trapping is broken. This phenomenon is called the overstable libration and Goldreich \& Schlichting (2014) is derived the condition required for the instability to grow. Substituting Eqs. (15) and (23) into Eq. (28) of Goldreich \& Schlichting (2014), we obtain the breaking condition for resonant trapping due to overstable libration under the aerodynamic gas drag as:

$3.29\left(\frac{j}{j-1}\right)^{3 / 2} \frac{M_{\mathrm{p}}}{M_{\mathrm{s}}}<\frac{\tau_{\text {aero }, 0}}{\tau_{\text {tide }, a}}$

Even once this condition is satisfied, it takes a time of $\sim \tau_{\text {aero,e }}$ for the instability to grow (Goldreich \& Schlichting 2014). If $\tau_{\text {aero, } e}>\tau_{\text {tide }, a}$, the trapped planetesimal is shepherded into the inner disk before escaping the resonance. The second condition required for the overstable libration is $\tau_{\text {aero, } e}<\tau_{\text {tide, } a}$, which one can write as:

$\frac{\tau_{\text {aero }, 0}}{\tau_{\text {tide }, a}}<0.54(j-1)^{-1 / 2}$,

using Eqs. (15) and 23.

\subsection{Required condition for the Accretion Sweet Spot}

The required condition for the SSP is summarised as:

$\max \left\{\begin{array}{l}1.54(j-1) e_{\text {cross }}{ }^{3} \\ 3.29\left(\frac{j}{j-1}\right)^{3 / 2} \frac{M_{\mathrm{p}}}{M_{\mathrm{s}}}\end{array}\right\}<\frac{\tau_{\text {aero }, 0}}{\tau_{\text {tide }, a}}<0.54(j-1)^{-1 / 2}$

The inner edge of the SSP is regulated by the aerodynamic shepherding and resonant shepherding. The dominating process depends on the planetary mass. On the other hand, the outer edge of SSP is regulated by the resonant shepherding only.

To derive above equations, we assume that $\tau_{\text {aero }, a} \gg \tau_{\text {tide, } a}$. Using the equilibrium eccentricity, we transform this condition as

$$
\frac{2.18 \eta_{\mathrm{gas}}{ }^{3 / 2}}{\sqrt{j-1}} \ll \frac{\tau_{\text {aero }, 0}}{\tau_{\text {tide }, a}} .
$$

In the nominal disk model, $\eta_{\text {gas }}$ is the order of $10^{-3}$ and this condition is easily achieved in the SSP. Thus, the eqs. (32) can be used for small planetesimals as long as the gas drag strength is scaled by eq. (16). We find that the location of the SSP is determined by the ratio of the gas drag damping timescale $\tau_{\text {aero, } 0}$ and the planetary migration timescale $\tau_{\text {tide, } a}$. We therefore conclude that our theoretical prediction is robust (and rather general) since the derivation of this condition does not depend on the assumed disk and migration models. As a result, Eq. (32) can be generally applied to various models of planetesimal accretion.

\section{Comparison with numerical results}

In this section we validate the derived equation for the SSP by comparison with numerical results. To that end, we perform $N$ body simulations similar to those presented in Shibata et al. (2020). We adopt different values of model parameters from those in Shibata et al. (2020) in order to identify whether the $\mathrm{SSP}$ is indeed regulated by the ratio of the two timescales, $\tau_{\text {aero, } 0}$ and $\tau_{\text {tide, } a}$.

\subsection{Numerical Model and Settings}

We consider the following scenario: Gas accretion has terminated and the young giant planet no longer grows in mass. The planet then migrates radially inward from a given semi-major axis within a protoplanetary disk. Initially there are many singlesized planetesimals interior to the planet's orbit. The migrating planet then encounters these planetesimals and captures some of them. The planetesimals are represented by test particles and, therefore, are affected only by the gravitational forces from the central star and planet, and the drag force by the disk gas. Here, we summarise our numerical model. The details of our numerical model are described in Appendix A

We assume that the planetary migration timescale is given by:

$\tau_{\text {tide }, a}=\tau_{\text {tide }, 0}\left(\frac{a_{\mathrm{p}}}{1 \mathrm{AU}}\right)^{1 / 2}$,

where $\tau_{\text {tide, } 0}$ is a scaling parameter. For the drag force by the disk gas, we adapt a model by Adachi et al. (1976). The gas drag force depends on the size of planetesimal $R_{\mathrm{pl}}$ and the profile of ambient disk gas. To focus on the dynamic process, we adopt simply the minimum-mass solar nebula model (Hayashi 1981) as our gas disk model. The surface density $\Sigma_{\text {gas }}$ and the disk' temperature $T_{\text {disk }}$ are given by:

$$
\begin{aligned}
\Sigma_{\text {gas }} & =\Sigma_{\text {gas }, 0}\left(\frac{r}{1 \mathrm{AU}}\right)^{-\alpha_{\text {disk }}}, \\
T_{\text {disk }} & =T_{\text {disk }, 0}\left(\frac{r}{1 \mathrm{AU}}\right)^{-2 \beta_{\text {disk }}},
\end{aligned}
$$

where $r$ is the radial distance from the initial mass centre of the star-planet system, $\Sigma_{\text {gas }, 0}=1.7 \times 10^{3} \mathrm{~g} / \mathrm{cm}^{2}, \alpha_{\text {disk }}=3 / 2, T_{\text {disk }, 0}=$ $280 \mathrm{~K}$ and $\beta_{\text {disk }}=1 / 4$. The protoplanetary disk is assumed to be vertically isothermal.

For the planetesimals we adopt a simple surface density profile given by:

$\Sigma_{\text {solid }}=Z_{\mathrm{s}} \Sigma_{\text {gas }, 0}\left(\frac{r}{1 \mathrm{AU}}\right)^{-\alpha_{\text {disk }}^{\prime}}$,

where $Z_{\mathrm{s}}$ is the solid-to-gas ratio (or the metallicity) and $\alpha_{\text {disk }}^{\prime}=$ $\alpha_{\text {disk }}=3 / 2$. To speed up the numerical integration, we follow the orbital motion of super-particles, each of which contains several equal-size planetesimals. The super-particles are initially distributed in $a_{\mathrm{pl} \text {,in }}<r<a_{\mathrm{pl} \text {,out }}$ with $a_{\mathrm{pl} \text {,in }}=0.3 \mathrm{AU}$ and $a_{\mathrm{pl} \text {,out }}=20 \mathrm{AU}$. The planet is initially located in such a way that the inner boundary of the feeding zone is consistent with the outer edge of the planetesimals disk, namely:

$a_{\mathrm{p}, 0}=\frac{a_{\mathrm{pl}, \mathrm{out}}}{1-2 \sqrt{3} h}$.

The calculation is artificially stopped once the planet reaches the orbit of $a_{\mathrm{p}, \mathrm{f}}=0.5 \mathrm{AU}$. First, we investigate the planetesimal accretion in a reference case, where $\tau_{\text {tide }, 0}=1 \times 10^{5} \mathrm{yr}$, 


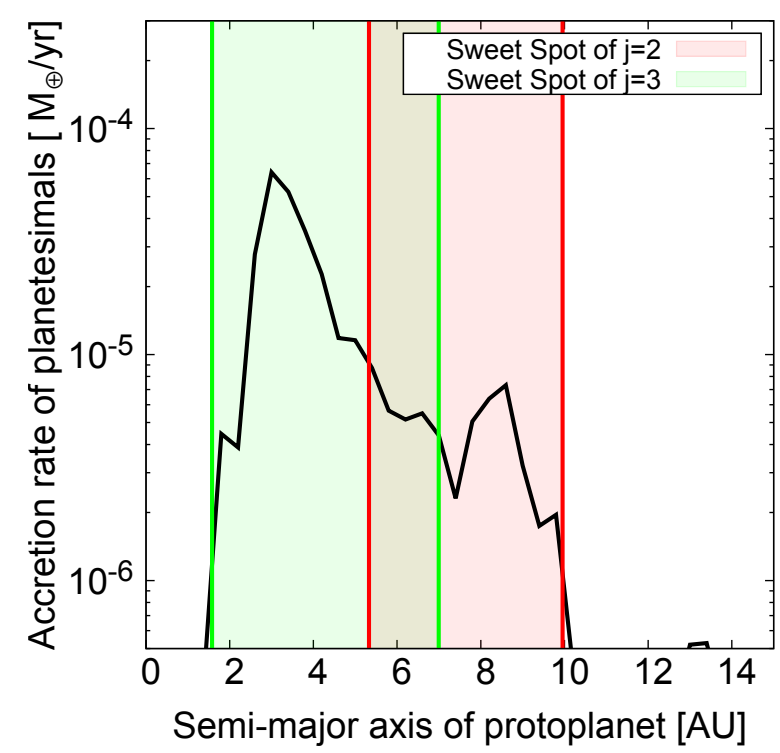

Fig. 4. The planetesimal accretion rate vs. the semi-major axis of the migrating planet. The vertical solid lines show the boundaries of the sweet spot for planetesimal accretion (SSP) given by Eq. [32. The red lines are for the case of $j=2$ and the green lines are for $j=3$. The filled areas show the SSP.

$R_{\mathrm{pl}}=1 \times 10^{7} \mathrm{~cm}$ and $M_{\mathrm{p}}=1 \times 10^{-3} M_{\mathrm{s}}$. In the reference case, the total amount of planetesimals are $M_{\text {tot }} \sim 43 M_{\oplus}$. The choices of the parameter values for the reference model are summarised in Table 2. We perform a parameter study for various values of $\tau_{\text {tide, }, 0}, R_{\mathrm{pl}}$, and $M_{\mathrm{p}}$. The dynamical integration for the planetesimals is performed using the numerical simulation code developed by Shibata \& Ikoma (2019). In this code, we integrate the equation of motion using the forth-order-Hermite integration scheme (Makino \& Aarseth 1992). For the timesteps, we adopt the method of Aarseth (1985).

\subsection{Comparison with the numerical result}

Figure 4 shows the planetesimal accretion rate at each semimajor axis of the migrating protoplanet, obtained in the simulation for the reference model. Planetesimal accretion is found to occur efficiently in a region of $2 \mathrm{AU} \lesssim a_{\mathrm{p}} \lesssim 10 \mathrm{AU}$. Beyond $10 \mathrm{AU}$, planetesimals are trapped in the mean motion resonances of $j=2$ and 3 with the migrating protoplanet. Our analysis of the numerical results shows that the condition of overstable libration is achieved, but the timescale of the instability growth is longer than the migration timescale; thus, the trapped planetesimals are shepherded without being captured by the protoplanet. Around $\sim 10 \mathrm{AU}$, planetesimals trapped in the $2: 1$ mean motion resonance start to escape due to the overstable libration. The large amount of shepherded planetesimals are supplied into the planetary feeding zone, so that the accretion rate peaks at around $\sim 8 \mathrm{AU}$. Aerodynamic shepherding starts around 5AU for the planetesimals in the $2: 1$ mean motion resonance, halting the supply of planetesimals through the $2: 1$ mean motion resonance and reducing the accretion rate significantly. Instead, planetesimal supply resumes through the $3: 2$ mean motion resonance around $7 \mathrm{AU}$ and the accretion rate increases again. More specifically, planetesimals that remain to be brought into the feeding zone through $2: 1$ mean motion resonance move to

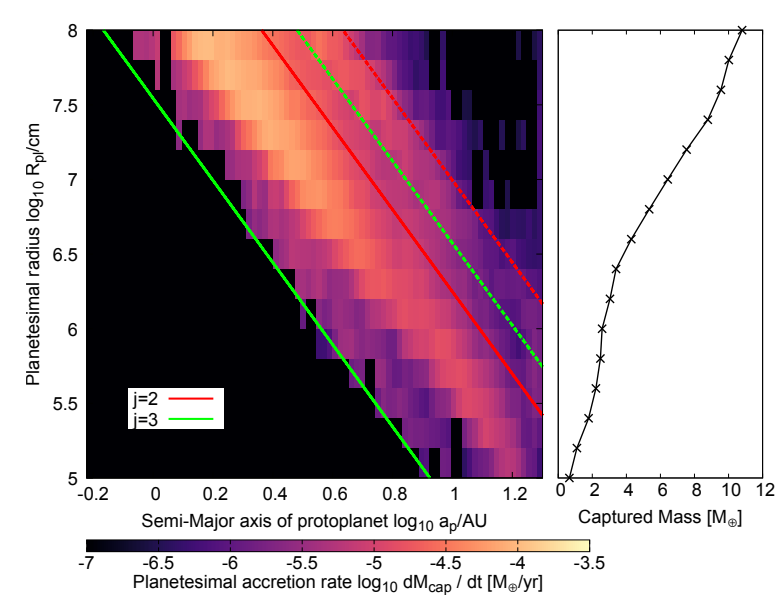

Fig. 5. The results of the parameter study regarding the size of planetesimals $R_{\mathrm{pl}}$. Left: Planetesimal accretion rate shown as a color contour on a plane of the semi-major axis of the migrating protoplanet and the planetesimal's radius. The analytically predicted inner and outer boundaries of the sweet spot are indicated with solid and dashed lines, respectively. The red lines are for $j=2$ and the green lines are for $j=3$. Right: Total mass of captured planetesimals as a function of the planetesimal radius.

the $3: 2$ resonance; namely, the accretion band shifts from $j=2$ to $j=3$. The accretion rate peaks again around $3 \mathrm{AU}$ and then rapidly decreases around $2 \mathrm{AU}$ due to aerodynamic shepherding.

In the simulation setting, the timescale ratio is given by:

$$
\begin{aligned}
\frac{\tau_{\text {aero }, 0}}{\tau_{\text {tide }, a}} & =\left|\frac{\tau_{\text {aero }, 0}}{\tau_{\text {tide }, a}}\right|_{1 \mathrm{AU}}\left(\frac{j-1}{j}\right)^{1 / 3} a^{11 / 4}, \\
& =\left|\frac{\tau_{\text {aero }, 0}}{\tau_{\text {tide }, a}}\right|_{1 \mathrm{AU}}\left(\frac{j-1}{j}\right)^{13 / 6} a_{\mathrm{p}}^{11 / 4},
\end{aligned}
$$

where

$$
\left|\frac{\tau_{\text {aero }, 0}}{\tau_{\text {tide }, a}}\right|_{1 \mathrm{AU}}=4.2 \times 10^{-3}\left(\frac{R_{\mathrm{pl}}}{10^{7} \mathrm{~cm}}\right)\left(\frac{\tau_{\text {tide }, 0}}{10^{5} \mathrm{yr}}\right)^{-1} .
$$

The red-shaded and green-shaded areas in Fig. 4 show the predicted regions of the SSP, $5 \mathrm{AU} \lesssim a_{\mathrm{p}} \lesssim 10$ AU for $j=2$ and $1 \mathrm{AU} \lesssim a_{\mathrm{p}} \lesssim 7 \mathrm{AU}$ for $j=3$, respectively, which one obtains using Eqs. (40) and (41) in Eq. (32). Those regions turn out to cover the areas where the accretion rate is higher than $10^{-6} M_{\oplus} / y r$. Thus, we conclude that the derived equation for the SSP reproduces well the numerical results in the reference model.

\subsection{Results of parameter studies}

As seen in Eq. (32), the location of the SSP depends on the ratio of the gas drag damping timescale $\tau_{\text {aero, } 0}$ to the planetary migration timescale $\tau_{\text {tide, } a}$ and the planetary mass $M_{\mathrm{p}}$. To investigate this dependence, we perform parameter studies for the size of planetesimals $R_{\mathrm{pl}}$, which determines $\tau_{\text {aero, } 0}$, the scaling factor of migration timescale $\tau_{\text {tide, } 0}$, and the planetary mass $M_{\mathrm{p}}$.

\subsubsection{Dependence on the planetesimal's size}

Since the gas drag damping timescale $\tau_{\text {aero, } 0}$ increases with the size of planetesimals, the location of the SSP is predicted to 


\begin{tabular}{llll}
\hline$M_{\mathrm{s}}$ & Mass of central star & 1.0 & $M_{\odot}$ \\
$Z_{\mathrm{s}}$ & Metallicity of central star & 0.018 & - \\
$M_{\mathrm{p}}$ & Mass of planet & 1.0 & $M_{\mathrm{Jup}}$ \\
$a_{\mathrm{p}, 0}$ & Initial semi-major axis of planet & eq. $[38$ & \\
$a_{\mathrm{p}, \mathrm{f}}$ & Final semi-major axis of planet & 0.5 & $\mathrm{AU}$ \\
$\tau_{\text {tide, } 0}$ & Scaling factor of migration timescale & $1.0 \times 10^{5}$ & $\mathrm{yr}$ \\
$\Sigma_{\text {disk,0}}$ & Surface density of disk gas at 1AU & $1.7 \times 10^{3}$ & $\mathrm{~g} / \mathrm{cm}^{2}$ \\
$T_{\text {disk,0}}$ & Temperature at 1AU & 280 & $\mathrm{~K}$ \\
$\alpha_{\text {disk }}$ & Exponent of disk gas profile & $3 / 2$ & - \\
$\beta_{\text {disk }}$ & Exponent of disk temperature profile & $1 / 4$ & - \\
$R_{\mathrm{pl}}$ & Radius of planetesimal & $1.0 \times 10^{7}$ & $\mathrm{~cm}$ \\
$a_{\mathrm{pl}, \text { in }}$ & Inner edge of planetesimal disk & 0.3 & $\mathrm{AU}$ \\
$a_{\mathrm{pl}, \text { out }}$ & Outer edge of planetesimal disk & 20 & $\mathrm{AU}$ \\
$\rho_{\mathrm{pl}}$ & Mean density of planetesimal & 2.0 & $\mathrm{~g} \mathrm{~cm}$ \\
$N_{\mathrm{sp}}$ & Initial number of super-particles & 12000 & - \\
$C_{\mathrm{d}}$ & Non-dimensional drag coefficient & 1 & - \\
\hline
\end{tabular}

Table 2. Parameters used in the reference model.

be closer to the central star for larger planetesimals (see, e.g., Eqs. (40) and (41)). We perform the numerical simulations, changing the size of planetesimals from $1 \times 10^{5} \mathrm{~cm}$ to $1 \times 10^{8} \mathrm{~cm}$ (or $\sim 0.01 M_{\oplus}$ ). Note that the assumption that mutual gravitational interaction among planetesimals is negligible is not valid for large planetesimals with sizes of $R_{\mathrm{pl}} \sim 10^{8} \mathrm{~cm}$. Nevertheless, a study for a wide parameter range is useful to deepen the physical understanding of the effect of the damping timescale on planetesimal accretion.

Figure 5 shows the results of the parameter study when changing the planetesimal's radius $R_{\mathrm{pl}}$. In the left panel, the planetesimal accretion rate is shown as a color contour on a plane of the semi-major axis of the migrating protoplanet and the planetesimal's radius. The analytically predicted inner and outer boundaries of the SSP derived in sec. 2.3 are shown with the solid and dashed lines, respectively. The red lines are for $j=2$ and the green lines are for $j=3$. In the right panel, the total mass of captured planetesimals $M_{\text {cap,tot }}$ is plotted as a function of the planetesimal's radius.

The location of the SSP (i.e., the region of high accretion rates) is found to be well reproduced by the analytical expressions derived in sec. 2.3. The maximum accretion rate and the total captured heavy-element mass are higher for larger planetesimals. This is because as the SSP moves inward, the amount of planetesimals shepherded into the SSP increases. More planetesimals are shepherded into the SSP when the planetesimals are larger, where the SSP locates farther from the initial planet location (or closer to the central star).

\subsubsection{Dependence on the planetary migration timescale}

Next, we perform a parameter study when changing the planetary migration timescale. Our analytic consideration above indicates that the location of the SSP is closer to the central star for faster planetary migration. We perform numerical simulations with various migration timescales by changing the scaling factor of migration timescale $\tau_{\text {tide }, 0}$ from $1 \times 10^{4} \mathrm{yr}$ to $1 \times 10^{6} \mathrm{yr}$.

Figure 6 shows the dependence of the results to the migration timescale $\tau_{\text {tide, } 0}$. Indeed, in the left panel, the SSP is found to be closer to the star for faster planetary migration (or smaller $\left.\tau_{\text {tide }, 0}\right)$. Since the SSP moves inward as $\tau_{\text {tide }, 0}$ decreases, for the same reason described above, the total mass of captured planetesimals $M_{\text {cap,tot }}$ increases with decreasing $\tau_{\text {tide, }, 0}$, which is confirmed in the right panel. The right axis shows $\tau_{\text {aero, } 0} / \tau_{\text {tide, } a}$ at

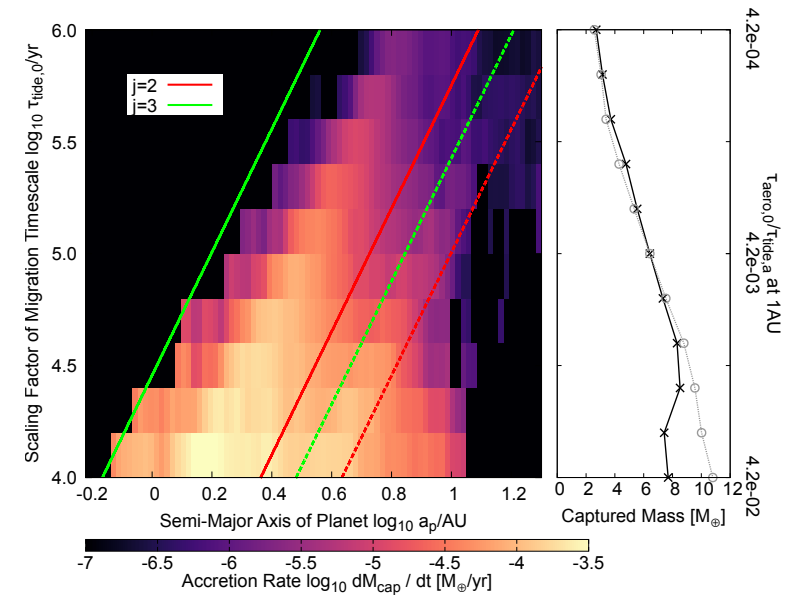

Fig. 6. Same as Fig. 5 but for the results of the parameter study regarding the migration timescale $\tau_{\text {tide, } 0}$. The gray circles in the right panel show the results of the parameter study regarding the size of planetesimals $R_{\mathrm{pl}}$ for comparison using the fraction of timescales $\tau_{\text {aero, } 0} / \tau_{\text {tide }, a}$ in vertical axis.

1AU calculated with Eq. (41). For comparison, we also plot with grey circles $M_{\text {cap,tot }}$ vs. $\tau_{\text {aero }, 0} / \tau_{\text {tide }, a}$ relation by converting the result shown in the right panel of Fig. 5 when using Eq. (41). Except for $\tau_{\text {tide, } 0} \lesssim 10^{4.6} \mathrm{yr}$, the two results are similar. This confirms that the location of the SSP scales with the ratio of the timescales $\tau_{\text {aero, } 0} / \tau_{\text {tide,a }}$.

The effects of planetary migration, however, seems somewhat more complicated relative to the dependence on the planetesimal's radius. First, as shown in the left panel, for relatively fast migration $\left(\tau_{\text {tide, } 0} \lesssim 10^{4.6} \mathrm{yr}\right)$, the outer boundary of the SSP expands beyond the one inferred analytically. Second, the $M_{\text {cap,tot }}$ Vs. $\tau_{\text {aero, } 0} / \tau_{\text {tide, } a}$ relation differs between the cases when we vary $R_{\mathrm{pl}}$ and varying $\tau_{\text {tide, } 0}$ for $\tau_{\text {tide, } 0} \lesssim 10^{4.6} \mathrm{yr}$. This is related to the stability of resonant trapping. As shown in sec. 2.2. the condition required for resonant trapping is that the timescale for planetesimals to cross the mean motion resonance is longer than the libration timescale. Using Eq. (10), we find that stable resonant trapping is difficult for fast planetary migration like $\tau_{\text {tide }, 0} \lesssim 10^{4}$ yr.

Figure 7 shows the changes in the phase angle of a planetesimal initially located at $a_{0}=13.8 \mathrm{AU}$ in the cases of (a) 


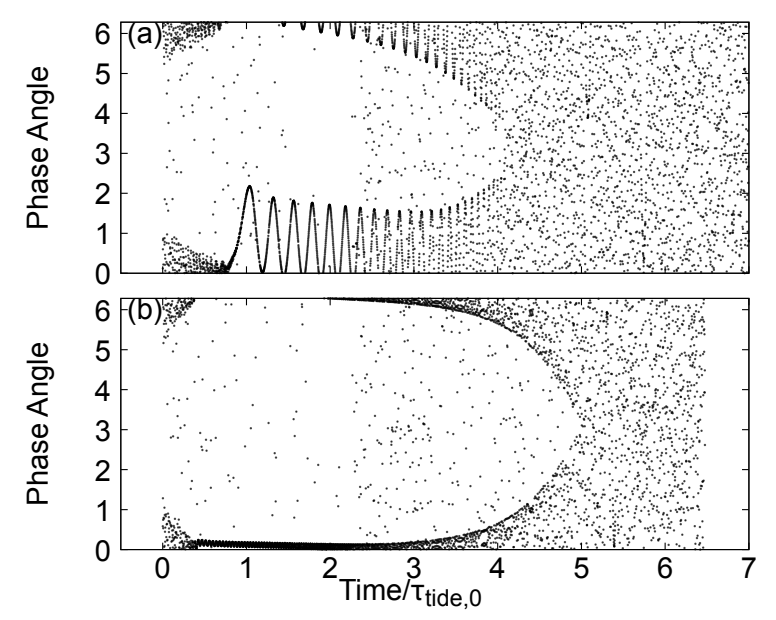

Fig. 7. Change in the phase angle of a planetesimal initially located at $a_{0}=13.8 \mathrm{AU}$. Panel (a) shows the case of $\tau_{\text {tide, } 0}=1 \times 10^{4} \mathrm{yr}$ and panel (b) shows the case of $\tau_{\text {tide }, 0}=1 \times 10^{5} \mathrm{yr}$. In panel (a), the phase angle oscillates indicating that the resonant trapping is unstable due to the fast planetary migration. Then, the resonant trapping is broken earlier in the case of panel (a) than that shown in panel (b).

$\tau_{\text {tide }, 0}=10^{4} \mathrm{yr}$ and (b) $\tau_{\text {tide }, 0}=10^{5} \mathrm{yr}$. In panel (a), the phase angle continues libration even after trapped into the resonance. This is because fast planetary migration perturbs the orbit of the trapped planetesimal and accelerates the overstable libration. The resonant trapping is, thus, broken earlier in the case of $\tau_{\text {tide }, 0}=10^{4} \mathrm{yr}$ than in the case of $\tau_{\text {tide }, 0}=10^{5} \mathrm{yr}$, which results in the outward expansion of the SSP. However, such an outward shift of the outer edge of the SSP does not lead to an increase in the mass of captured planetesimals. This is because fast planetary migration breaks the resonant trapping as soon as the trapped planetesimal reaches the equilibrium condition. The planetesimal in panel (a) escapes from the resonant trapping with $e \sim 0.7$ and that in panel (b) with $e \sim 0.4$. The capture probability of planetesimals decreases with increasing eccentricity (Ida \& Nakazawa 1989; thus, the total mass of captured planetesimals does not increase with the expansion of the SSP in the cases of $\tau_{\text {tide }, 0} \lesssim 10^{4.6} \mathrm{yr}$.

\subsubsection{Dependence on the planetary mass}

Finally, we perform a parameter study of the simulations when varying the mass of the migrating protoplanet $M_{\mathrm{p}}$ in the range of $M_{\mathrm{p}} / M_{\mathrm{s}}$ between $1 \times 10^{-4}$ and $1 \times 10^{-2}$. The results are summarised in Figure 8 We find that the semi-major axis of the outer boundary of the SSP is insensitive to the protoplanet mass, which is consistent with $\mathrm{Eq}(32)$. In this figure we indicate the locations of the inner edge of the SSP for $j=4$ and 5 in addition to those for $j=2$ and 3 given by Eq. 32, confirming that Eq. (32) accuratly reproduces the numerical result. As shown in Fig. 3, the crossing points between the resonance centre and the feeding zone boundary disappear once the planetary mass exceeds a specific value. In Fig. 8, we show the $M_{\mathrm{p}}-a_{\mathrm{p}}$ relation corresponding to the disappearance of the crossing points with gray solid lines. This is also consistent with the numerical result.

As found in Shibata et al. (2020), the total mass of captured planetesimals $M_{\text {cap,tot }}$ changes with the planetary mass in a nonmonotonic manner and takes local maxima at $M_{\mathrm{p}} / M_{\mathrm{s}} \sim 10^{-3.6}$, $10^{-3.3}, 10^{-2.7}$ and $10^{-2.2}$. This periodical changes come from

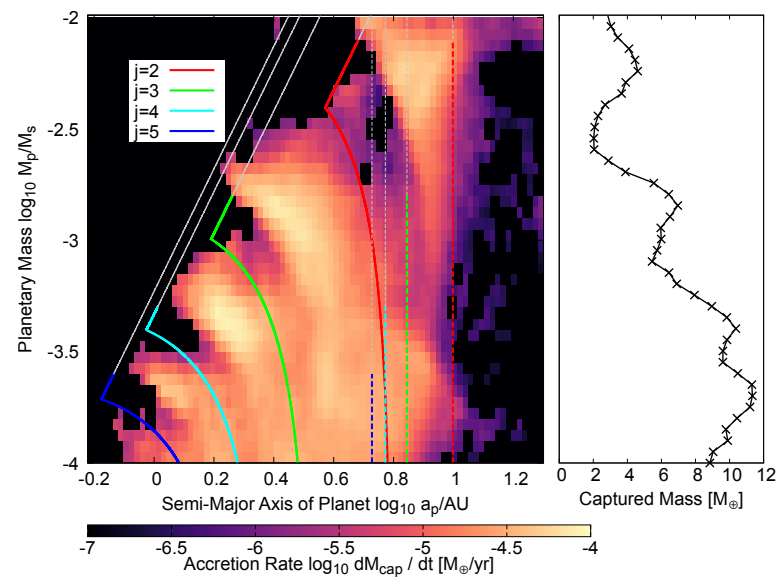

Fig. 8. Same as Fig. 5. but for the results of the parameter study when varying the protoplanet's mass $M_{\mathrm{p}}$. The red, green, skyblue, and blue lines represent the SSP inner (solid lines) and outer (dashed lines) boundaries determined by the resonant trapping, which is predicted by Eq. (32) for $j=2,3,4$, and 5, respectively. The coloured lines change into the gray lines once the protoplanet mass exceeds a threshold value, beyond which the accretion bands disappear (see Fig. 3.

the corresponding shifts of the inner boundary of the SSP. The shepherding process that regulates the inner boundary of the SSP changes from the aerodynamic shepherding to the resonant shepherding with increasing planetary mass. The accretion bands also change with the protoplanet mass. Because of these effects, the location of the inner boundary of the SSP shifts nonmonotonically which results in the non-monotonic change of $M_{\text {cap,tot }}$. The global decreasing trend with increasing protoplanet mass comes from the outward shift of the inner boundary.

\section{Discussion}

\subsection{Collision and ablation of highly-excited planetesimal}

Our model neglects the effect of planetesimal collisions. During the planetary migration, planetesimals are swept by the resonances and the enhanced local density of planetesimals increases the frequency of planetesimal collisions, which might initiate the collisional cascade (Batygin \& Laughlin 2015). Collisional cascade (e.g. Dohnanyi 1969) changes the size distribution of planetesimals and the perturbation adding on the planetesimal orbits by the collisions would also affect the resonant configuration (Malhotra 1993b). In this section, we consider the effect of planetesimal collisions on the location of the SSP and the planetesimal accretion.

\subsubsection{Collision timescale of shepherded planetesimals}

First, we estimate the collision timescale using the number density of planetesimals $n_{\mathrm{pl}}$, the collision cross section $\sigma$ and the relative velocity $u$. We consider a swarm of planetesimals that is trapped in mean motion resonances with total mass of $M_{\text {shep }}$. For simplicity, we consider that these planetesimals have the same mass. The planetesimals are distributed in the ring-like region with a width of $\sim 2 a\langle e\rangle$ and thickness of $\sim 2 a \tan \langle i\rangle$. The colli- 


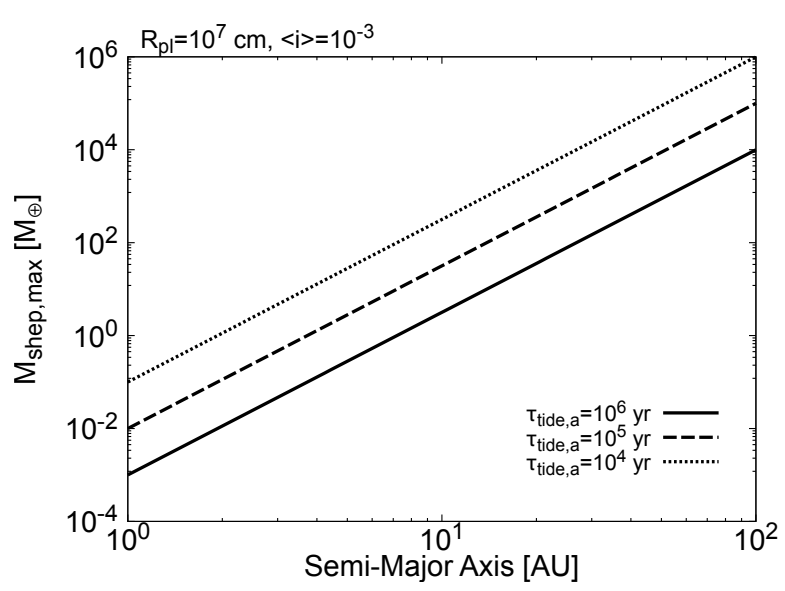

Fig. 9. Maximum mass of planetesimals that can be shepherded in the mean motion resonances without effective mutual collisions. We show the cases of $R_{\mathrm{pl}}=10^{7} \mathrm{~cm}$ and $\langle i\rangle=10^{-3}$. The solid, dashed, and dotted lines correspond to $\tau_{\text {tide }, a}=10^{6} \mathrm{yr}, 10^{5} \mathrm{yr}$ and $10^{4} \mathrm{yr}$, respectively.

sion timescale between these planetesimals $\tau_{\text {col }}$ is given as:

$$
\begin{aligned}
\tau_{\mathrm{col}} & =\left(n_{\mathrm{pl}} \sigma u\right)^{-1}, \\
& =\left(\frac{M_{\text {shep }}}{2 \pi a \cdot 2 a\langle e\rangle \cdot 2 a \tan \langle i\rangle} \cdot \pi R_{\mathrm{pl}}^{2} \cdot\langle e\rangle v_{\mathrm{K}}\right)^{-1}, \\
& =3 \times 10^{5} \mathrm{yr}\left(\frac{\mathrm{M}_{\mathrm{shep}}}{10 \mathrm{M}_{\oplus}}\right)^{-1}\left(\frac{\rho_{\mathrm{pl}}}{2 \mathrm{~g} / \mathrm{cm}^{3}}\right)\left(\frac{\mathrm{R}_{\mathrm{pl}}}{10^{7} \mathrm{~cm}}\right) \\
& \left(\frac{a}{10 \mathrm{AU}}\right)^{7 / 2}\left(\frac{\tan \langle i\rangle}{10^{-3}}\right)\left(\frac{M_{\mathrm{s}}}{M_{\odot}}\right)^{-1 / 2} .
\end{aligned}
$$

Here, we neglect the effect of gravitational focusing because of the high relative velocities of planetesimals. $M_{\text {shep }}$ increases as the protoplanet migrates and sweeps planetesimals, but decreases after reaching the SSP because the trapped planetesimals start to escape from the mean motion resonances. As suggested by eq. (44), $\tau_{\text {col }}$ rapidly decreases with the inward planetary migration. Planetesimals start to collide with each other where the collision timescale $\tau_{\text {col }}$ is comparable to the migration timescale $\tau_{\text {tide, } a}$. The maximum mass of planetesimals shepherded into the SSP without active planetesimal collisions $M_{\text {shep,max }}$ is estimated as:

$$
\begin{aligned}
& M_{\text {shep,max }}=30 M_{\oplus}\left(\frac{\tau_{\text {tide }, a}}{10^{5} \mathrm{yr}}\right)^{-1}\left(\frac{\rho_{\mathrm{pl}}}{2 \mathrm{~g} / \mathrm{cm}^{3}}\right)\left(\frac{R_{\mathrm{pl}}}{10^{7} \mathrm{~cm}}\right) \\
&\left(\frac{a}{10 \mathrm{AU}}\right)^{7 / 2}\left(\frac{\tan \langle i\rangle}{10^{-3}}\right)\left(\frac{M_{\mathrm{s}}}{M_{\odot}}\right)^{-1 / 2} \cdot
\end{aligned}
$$

Figure 9 shows $M_{\text {shep,max }}$ as a function of the radial distance from the central star. During the planetary migration, despite the increase of $M_{\text {shep }}, M_{\text {shep,max }}$ rapidly decreases. We conclude that the trapped planetesimals collide with each other during the planetary migration phase if the protoplanet migrates a long distance in the radial direction before entering the SSP.

\subsubsection{Collisional cascade in the resonant shepherding}

The outcome of planetesimal collision is determined by the specific energy, which is the kinetic energy of impacting planetesimal normalised by the mass of impacted planetesimal, given by (e.g. Armitage 2010):

$Q=1.1 \times 10^{12} \mathrm{erg} / \mathrm{g}\left(\frac{e}{0.5}\right)^{2}\left(\frac{R_{\mathrm{im}}}{R_{\mathrm{pl}}}\right)^{3}\left(\frac{M_{\mathrm{s}}}{M_{\odot}}\right)\left(\frac{a}{10 \mathrm{AU}}\right)^{-1}$,

where $R_{\mathrm{im}}$ is the radius of the impacting planetesimal. The critical value for shattering planetesimals can be scaled as:

$$
Q_{\mathrm{D}}^{*}=q_{\mathrm{s}}\left(\frac{R_{\mathrm{pl}}}{1 \mathrm{~cm}}\right)^{a}+q_{\mathrm{g}} \rho_{\mathrm{pl}}\left(\frac{R_{\mathrm{pl}}}{1 \mathrm{~cm}}\right)^{b} \text {. }
$$

Benz \& Erik (1999) and Leinhardt \& Stewart (2009) determined the values of fitting parameters in this equation. For icy planetesimals with an impact velocity of $3 \mathrm{~km} / \mathrm{s}$, they obtained $q_{\mathrm{s}}=1.6 \times 10^{7} \mathrm{erg} / \mathrm{g}, q_{\mathrm{g}}=1.2 \mathrm{erg} \mathrm{cm} / \mathrm{g}^{2}, a=-0.39$ and $b=1.26$. Using eq. (46) and (47), we find that km-sized planetesimals are shattered by the collisions of planetesimals larger than $R_{\mathrm{im}, \mathrm{th}}$, which is given as:

$$
\frac{R_{\mathrm{im}, \mathrm{th}}}{R_{\mathrm{pl}}}=0.13\left(\frac{R_{\mathrm{pl}}}{10^{7} \mathrm{~cm}}\right)^{0.42}\left(\frac{e}{0.5}\right)^{-2 / 3}\left(\frac{M_{\mathrm{s}}}{M_{\odot}}\right)^{-1 / 3}\left(\frac{a}{10 \mathrm{AU}}\right)^{1 / 3}
$$

We find that collisions of trapped planetesimals easily result in the dispersal and formation of many smaller planetesimals. The generated smaller planetesimals shortens the collisional timescale. Namely, during the planetary migration phase, $M_{\text {shep }}$ increases and once it exceeds the threshold value $M_{\text {shep,max }}$, the trapped planetesimals start to collide with each other and initiate collisional cascade. The planetesimal size distribution is largely changed and the major source of solid material would be the smaller planetesimals. As shown by our simulations, the decrease in planetesimal size shifts the location of the SSP outward. Therefore the possibility of collisional cascade occuring during planetary migration could affect the efficiency of planetesimal accretion.

The value of $M_{\text {shep,max }}$ after the initiation of the collisional cascade would be affected by the amount of dispersed planetesimals left in the mean motion resonances. Non-dispersed planetesimals with radii of $R_{\mathrm{pl}}$ can be supplied as the planet migrates, however, if a large amount of dispersed planetesimals larger than $R_{\text {im,th }}$ are left in the resonance, $M_{\text {shep,max }}$ is decreased by a factor of $\sim R_{\mathrm{im}, \mathrm{th}} / R_{\mathrm{pl}}$ at most. As we discuss below, impacted planetesimals might be removed from the mean motion resonances and small fragments generated by the collisional cascades would be drifted into the inner disk region faster than the planet. Thus, after the initiation of the collisional cascade, the size distribution of planetesimals in the resonant shepherding would be affected by various physical processes. We suggest that the mutual collisions of planetesimals in the resonant shepherding could be important for determining the population of small objects in planetary systems.

In the above discussion, we assumed that the relative velocity of planetesimals $u$ is approximated by $\sim e v_{\mathrm{K}}$. However, the alignment of planetesimal orbits via gravitational perturbation from the protoplanet is known to reduce the relative velocity of planetesimals. Guo \& Kokubo (2021) investigated the effect of orbital alignment due to the secular perturbation which converges the longitude of pericenter into a certain value; namely, planetesimal orbits are aligned on the inertial plane. Using N-body simulations, they found that the relative velocity of planetesimals are reduced outside the mean motion resonances. According to their results, orbital alignment is not effective inside the mean motion resonances because of the resonant perturbation. In the planetary migration phase, however, the resonance angle $\varphi$ converges into zero and planetesimal orbits can 

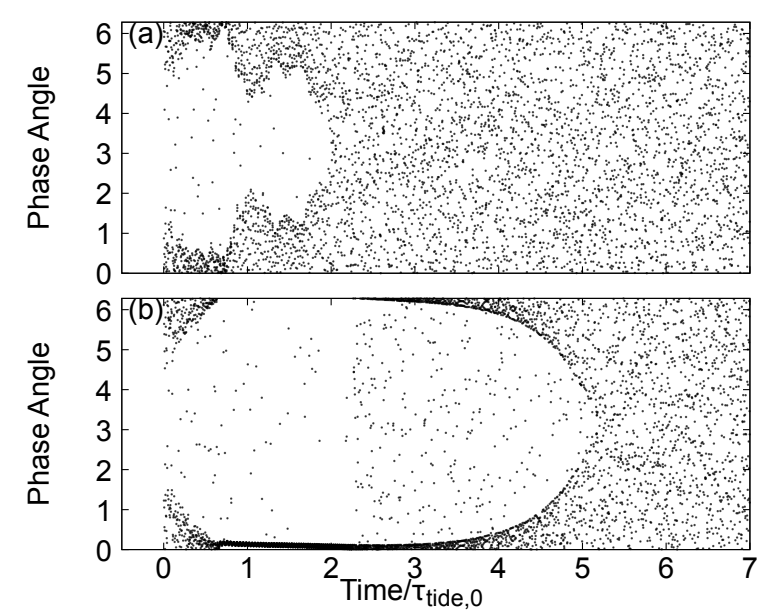

Fig. 10. Same as Fig. 7. but (a) for the case with artificial perturbation given by Eq. (50) and (b) without artificial perturbation. Here we use our reference model with $R_{\mathrm{pl}}=1 \times 10^{7} \mathrm{~cm}, \tau_{\text {tide, } 0}=1 \times 10^{5} \mathrm{yr}$ and $M_{\mathrm{p}} / M_{\mathrm{s}}=1 \times 10^{-3}$.

be aligned on the plane rotating with the protoplanet (see fig. $\mathrm{C} 1$ and $\mathrm{C} 2$ in Shibata et al. (2020)). In the ideal case, the relative velocity of these planetesimals is smaller than $\sim e v_{\mathrm{K}}$ and some collisions would result in sticking. In addition, the mean inclination of planetesimals $\langle i\rangle$ is difficult to estimate, but it is important for collision timescale. The viscous stirring between trapped planetesimals might affect the mean inclination of planetesimals $\langle i\rangle$, which is not considered in our simulations. Under effective viscous stirring, the velocity dispersion of the planetesimal swarm keeps a simple relation of $\left\langle e^{2}\right\rangle^{1 / 2} \sim 2\left\langle i^{2}\right\rangle^{1 / 2}$ (e.g. Ohtsuki et al. 2002). In this case, the collision timescale increases and $M_{\text {shep,max }}$ would be larger than indicated in fig. 9. However, it is still unclear whether the energy equipartition works in the resonant trapping where the planetesimal's eccentricity is excited by the protoplanet. It is clear that both $M_{\text {shep,max }}$ and the planetesimal size distribution depends on the interactions between planetesimals in the mean motion resonances. We suggest that this topic should be investigated in detail in future work.

\subsubsection{Breakup of resonant trap}

High-velocity collisions of planetesimals perturb the orbit of trapped planetesimals and break the resonant trapping. Using numerical simulations, Malhotra (1993b) found that for a planet of $10 M_{\oplus}$, resonant trapping is broken with a velocity kick of $\gtrsim 0.003 v_{\mathrm{K}}$. Therefore, once $M_{\text {shep }}$ exceeds the threshold value and the trapped planetesimals start to collide with each other, the resonant trapping can be broken by planetesimal collisions. However, the resonant width increases with increasing planetary mass (see Eq. (5); namely breaking the resonant trapping is more difficult for more massive planets) and not all high-velocity collisions lead to the breaking of resonant trapping (see Fig. $6 \mathrm{~b}$ of Malhotra (1993b)). In addition, as mentioned in the previous section, the alignment of the orbits by the resonant trapping might reduce the relative velocity of planetesimals. The possibility of resonant breaking by the planetesimal collisions need to be investigated in future work. Here, we consider the cases where the resonant trapping is broken by planetesimal collisions.

As shown in sec. 2, planetesimal accretion occurs only in a limited ring-like region within the protoplanetary disk. This

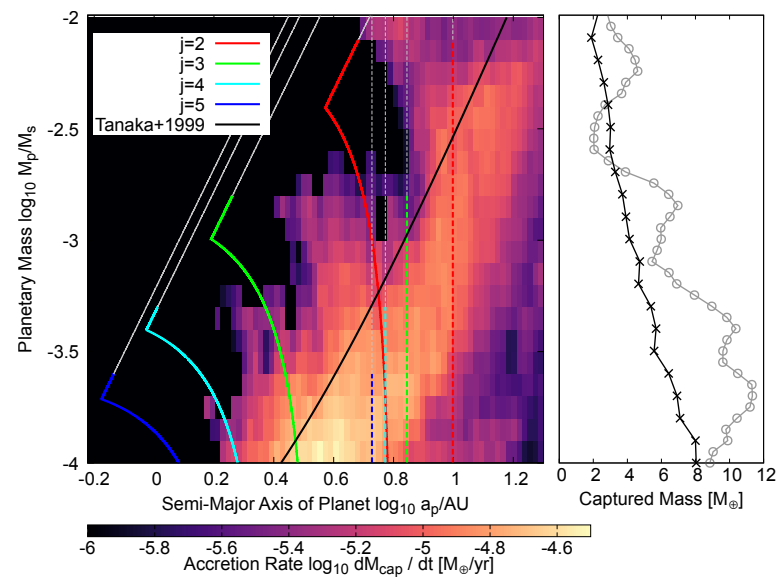

Fig. 11. Same as Fig. 5. but for the results of a parameter study for planetary mass $M_{\mathrm{p}}$ with the artificial perturbations (see Eq. (50)). In the right panel, the results of a parameter study for planetary mass $M_{\mathrm{p}}$ without the artificial perturbations are plotted with gray circles for comparison.

accretion process seems different from the one found for Earthmass planets in Tanaka \& Ida (1999). In their dynamical simulations, Tanaka \& Ida (1999) broke the resonant traps instantaneously by exerting artificial perturbations on the trapped planetesimals (see below), supposing that the planetesimals have their motion changed via the interactions with other planetesimals. Then, comparing the timescale for the protoplanet's migration with that for scattering of planetesimals by the migrating protoplanet, they derived a condition required for planetesimal accretion as:

$\tau_{\text {tide }, a}<0.81\left\{\sqrt{1+0.45\left(\frac{\tau_{\mathrm{aero}, 0}}{T_{\mathrm{K}, \mathrm{p}}}\right)^{2 / 3}}+1\right\}^{2} \frac{T_{\mathrm{K}, \mathrm{p}}}{h^{2}}$.

By contrast, when deriving Eq. (32) and also performing the dynamical simulations, we considered an extreme case without such modification to the planetesimals motion.

Using the artificial perturbation developed in Tanaka \& Ida (1997), we perform additional numerical simulations. We randomise the phase angle by adding displacements in orbital elements except $a, e$ and $i$. The size of the displacement is set as:

$\Delta \theta=2 \pi f_{\mathrm{p}}$,

where $f_{\mathrm{p}}$ is a scaling factor of perturbation strength and randomly given from -0.01 to 0.01 . We add $\Delta \theta$ to orbital angles (longitude of ascending node $\Omega$, longitude of pericentre $\varpi$ and mean longitude at epoch $\epsilon$ ) every conjunction time. Figure 10 shows the temporal change in the phase angle in the case with (a) and without (b) the artificial perturbations. As shown in panel (a), even under strong perturbations (corresponding to up to $1 \%$ displacement in the phase angle at every conjunction), the mean motion resonances with a Jupiter-mass planet are efficient in trapping planetesimals and, thereby, resonance shepherding occurs. This is because the resonance width (see Eq. (57) is larger for Jupiter-mass planets than that for Earth-mass planets. The perturbations is, however, found to hasten the breakup of resonant trapping, because resonant trapping is weakened and over-stable libration is accelerated.

Figure 11 shows the results of the parameter study when varying the planetary mass $M_{\mathrm{p}} / M_{\mathrm{s}}$ including the artificial perturbations given by Eq. (50). For comparison, we also show the 
results without artificial perturbations with open circles in the right panel. Due to the artificial perturbations, the SSP shifts outward (see the left panel) and the total mass of captured planetesimals is reduced (see the right panel). In the left panel, the black solid line shows the condition of shepherding obtained in Tanaka \& Ida (1999) (see eq. (49)); planetesimal accretion occurs in regions exterior to it. This black line corresponds to the inner boundary of the SSP for the case where resonant traps are perfectly broken. Although it might seem counter-intuitive, we conclude that without perturbations, resonant trapping shifts the SSP inward and enhances planetesimal accretion due to the effect of accretion bands.

\subsubsection{Ablation of eccentric planetesimals}

A highly-excited planetesimal has a high relative velocity compared to that of the disk gas. Strong gas drag from the gaseous disk (e.g., Pollack et al. 1986) and generated bow-shock (e.g., Tanaka et al. 2013) heats the planetesimal's surface and triggers the ablation of volatile materials. Planetesimals trapped in the mean motion resonances have eccentric orbits and feel strong gas drag, and therefore ablation is expected to be important. The ablation rate depends on the rate of heat transfer from the ambient disk gas $\Lambda$. We set $\Lambda$ to range between 0.003 and 0.6 for gas drag heating, and between $\sim 0.01$ and $\sim 0.1$ for bow shock heating (see Tanaka et al. 2013, and references therein). Recently Eriksson et al. (2021) explored the possibility of perfect ablation of planetesimals around a massive protoplanet using $\Lambda=C_{\mathrm{d}} / 4$, which is the upper limit of the heat transfer rate (D'Angelo \& Podolak 2015). According to their nominal model result, ablation of highly excited planetesimals is effective in the inner disk region $\lesssim 10 \mathrm{AU}$ and large amount of solid materials are ablated. The heating process strongly depends on the eccentricity of planetesimals. Using eqs. 24) and (32), we find that the eccentricity of planetesimals inside the SSP decreases from $\sim 0.5$ to 0.1 . To avoid effective ablation planetesimals must enter the SSP in the outer disk region $\gtrsim 10 \mathrm{AU}$. If the secular resonance triggered by the disk's gravity is effective, the ablation would be effective in more outer disk region (Nagasawa et al. 2019). On the other hand, if $\Lambda$ is much smaller than $C_{\mathrm{d}} / 4$, the ablation of planetesimals would be inefficient.

The investigation presented above concerning planetesimal collision and ablation implies that planetesimal accretion is favoured in the outer disk region $\gtrsim 10 \mathrm{AU}$. Detailed future studies that focus on collision and ablation processes are clearly desirable.

\subsection{The case of Type II migration with shallow-gap}

In the classical picture of the Type II migration (e.g. Lin \& Papaloizou 1993), a giant planet migrates with a deep gap opened in the vicinity of its orbit in a gaseous protoplanetary disk. For an extremely deep gap, the radial gas flow toward the central star is stemmed completely by the gap and, consequently, the planet migrates with the gaseous disk contracting via viscous diffusion. Recent hydrodynamic simulations, however, reveal that the gap bottom is not so deep and the gas can cross the gap (Kanagawa et al. 2015, 2016, 2017). In this case, the planetary migration is not in the classical regime; instead, the torque exerted on the planet is given similarly to that in the Type I regime (Kanagawa et al. 2018). Thus, the migration timescale in the new type II regime is written as:

$\tau_{\text {tide }, a, \mathrm{II}} \sim \frac{1}{2 c}\left(\frac{M_{\mathrm{p}}}{M_{\mathrm{s}}}\right)^{-1}\left(\frac{r_{\mathrm{p}}{ }^{2} \Sigma_{\text {gap }}}{M_{\mathrm{s}}}\right)^{-1}\left(\frac{h_{\mathrm{s}}}{r_{\mathrm{p}}}\right)^{2} \Omega_{\mathrm{K}}{ }^{-1}$,

where $c$ is a constant of the order of unity and $\Sigma_{\text {gap }}$ is the surface density of disk gas at the gap bottom; $\Sigma_{\text {gap }}$ depends on the unperturbed surface density of disk gas $\Sigma_{\text {gas }}$ and is given from the hydrodynamic simulations of Kanagawa et al. (2017) as:

$\Sigma_{\text {gap }}=\frac{\Sigma_{\text {gas }}}{1+0.04 K}$

with

$K=\left(\frac{M_{\mathrm{p}}}{M_{\mathrm{s}}}\right)^{2}\left(\frac{h_{\mathrm{s}}}{r_{\mathrm{p}}}\right)^{-5} \alpha_{\mathrm{vis}}{ }^{-1}$,

where $\alpha_{\text {vis }}$ is the alpha parameter for disk gas turbulent viscosity (Shakura \& Sunyaev 1973). From Eqs. (51) and (52), we obtain:

$\tau_{\text {tide }, a, \text { II }} \sim \frac{0.02}{c} \alpha_{\text {vis }}{ }^{-1}\left(\frac{r_{\mathrm{p}}{ }^{2} \Sigma_{\text {gas }}}{M_{\mathrm{p}}}\right)^{-1}\left(\frac{h_{\mathrm{s}}}{r_{\mathrm{p}}}\right)^{-3} \Omega_{\mathrm{K}}{ }^{-1}$,

for $1 \ll 0.04 K$ or

$M_{\mathrm{p}} \gg 8 M_{\oplus}\left(\frac{\alpha_{\mathrm{vis}}}{10^{-3}}\right)^{1 / 2}\left(\frac{r_{\mathrm{p}}}{1 \mathrm{AU}}\right)^{5\left(1-2 \beta_{\text {disk }}\right) / 4}$.

Here we discuss the location of SSP and planetesimal accretion onto the protoplanet migrating with eq. (54).

\subsubsection{The location of the accretion sweet spot}

The location of the SSP depends on the ratio of the planetary migration and aerodynamic gas drag timescales. The timescale of damping by aerodynamic gas drag $\tau_{\text {aero, } 0}$ in the disk mid-plane also depends on the disk structure (see Eq. (16) ) as:

$\tau_{\text {aero }, 0}=\frac{8 \sqrt{2 \pi}}{3 C_{\mathrm{d}}}\left(\frac{\Sigma_{\mathrm{gas}}}{\rho_{\mathrm{pl}} R_{\mathrm{pl}}}\right)^{-1} \frac{h_{\mathrm{s}}}{r_{\mathrm{pl}}} \Omega_{\mathrm{K}}^{-1}$.

Note that $\tau_{\text {tide, } a \text {,II }}$ is a function of $r_{\mathrm{p}}$, whereas $\tau_{\text {aero, } 0}$ is a function of $r_{\mathrm{pl}} ; r_{\mathrm{p}} / r_{\mathrm{pl}}=((j-1) / j)^{2 / 3}$ for a planetesimal in the $j: j-1$ mean motion resonance with the planet. In this case, Eq. 56 can be written as a function of $r_{\mathrm{p}}$ as:

$\tau_{\text {aero }, 0}=\frac{8 \sqrt{2 \pi}}{3 C_{\mathrm{d}}}\left(\frac{\Sigma_{\mathrm{gas}}}{\rho_{\mathrm{pl}} R_{\mathrm{pl}}}\right)^{-1} \frac{h_{\mathrm{s}}}{r_{\mathrm{p}}} \Omega_{\mathrm{K}}^{-1}\left(\frac{j-1}{j}\right)^{2 \gamma_{\mathrm{disk}} / 3}$,

with

$\gamma_{\text {disk }}=2+\alpha_{\text {disk }}-\beta_{\text {disk }}$

Here, we have assumed that the planetesimals trapped in mean motion resonances are outside the gap. If the disk viscosity is small, however, the gap slope reaches the locations of the resonances; in that case, the damping timescale increases a few times. For simplicity, we neglect this effect.

From Eqs. 54 and (57), the ratio of the two timescales is given by:

$\frac{\tau_{\text {aero }, 0}}{\tau_{\text {tide }, a \text { II }}}=1 \times 10^{-6} C^{-1}\left(\frac{M_{\mathrm{p}}}{M_{\mathrm{s}}}\right)^{-1}\left(\frac{r_{\mathrm{p}}}{1 \mathrm{AU}}\right)^{4\left(1-\beta_{\text {disk }}\right)}\left(\frac{j-1}{j}\right)^{2 \gamma_{\text {disk }} / 3}$, 


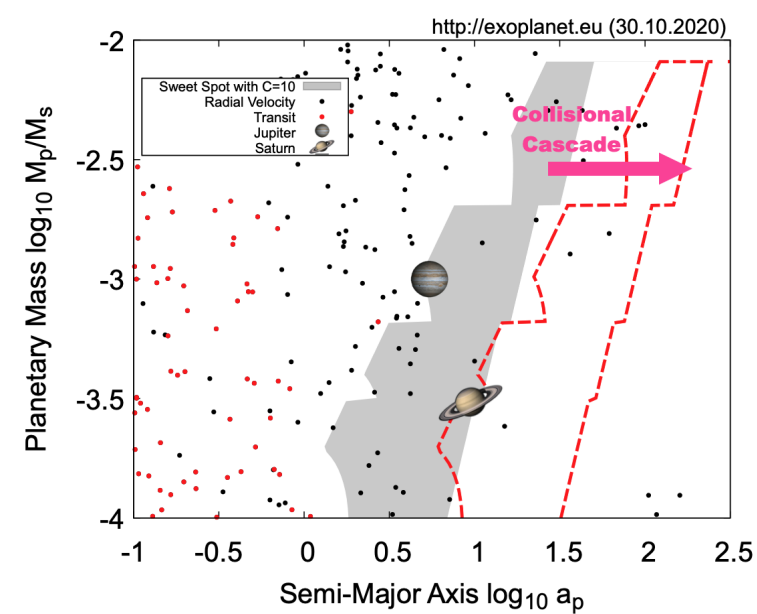

Fig. 12. Distribution of confirmed exoplanets in the semi-major axis vs. planetary mass plane (http://exoplanet.eu). The black points are exoplanets observed by the radial-velocity method. The red points are exoplanets observed by the radial-velocity + transit methods. As for masses of exoplanets indicated with the black points, the observe values of $M_{\mathrm{p}} \sin i$ are simply used. The gray area shows the theoretical sweet spot for planetesimal capture given by Eqs. (61) and $\sqrt{62}$ ) with $C=10$. The red dashed line shows the theoretical sweet spot for planetesimal capture with $C=10^{3}$, which corresponds to the case where collisional cascade broke planetesimals down to $R_{\mathrm{pl}} \lesssim 10^{5} \mathrm{~cm}$.

with

$C=\frac{2 C_{\mathrm{d}}}{c}\left(\frac{\alpha_{\mathrm{vis}}}{10^{-3}}\right)^{-1}\left(\frac{\rho_{\mathrm{pl}}}{2 \mathrm{~g} / \mathrm{cm}^{3}}\right)^{-1}\left(\frac{R_{\mathrm{pl}}}{10^{7} \mathrm{~cm}}\right)^{-1}\left(\frac{M_{\mathrm{s}}}{M_{\odot}}\right)\left(\frac{\left|h_{\mathrm{s}} / r\right|_{1 \mathrm{AU}}}{0.03}\right)^{-4}$,

Equation (59) indicates that the surface gas density profile only weakly affects the timescale ratio, which does not explicitly depend on $\Sigma_{\text {gas }}$ and weakly depends on $\alpha_{\text {disk }}$, namely, $((j-$ $1) / j)^{2 \alpha_{\text {disk }} / 3}$. This means that as the protoplanetary disk evolves, the timescale ratio hardly changes, suggesting that the location of the SSP is fixed.

Assuming $\alpha_{\text {disk }}=1$, which is valid in a steady viscous accretion disk except for its outermost region (e.g. Lynden-Bell \& Pringle 1974), and $\beta_{\text {disk }}=1 / 4$, which is valid in an optically thin disk, and substituting Eq. (59) into Eq. (32), we obtain the semimajor axes of the inner and outer edge of SSP,$a_{\mathrm{SS} \text {,in }}$ and $a_{\mathrm{SS} \text {,out }}$, respectively, as:

$$
\begin{aligned}
a_{\mathrm{SS}, \text { in }} & =\max \left\{\begin{array}{l}
1 C^{1 / 3}\left(\frac{M_{\mathrm{p}} / M_{\mathrm{s}}}{10^{-3}}\right)^{2 / 3}\left(\frac{j}{j-1}\right)^{10 / 9} \\
1 C^{1 / 3}\left(\frac{M_{\mathrm{p}} / M_{\mathrm{s}}}{10^{-3}}\right)^{1 / 3}\left\{\frac{j^{11}}{(j-1)^{5}}\right\}^{1 / 18} \frac{e_{\text {cross }}}{0.1}
\end{array}\right\} \mathrm{AU}, \\
a_{\mathrm{SS}, \text { out }} & =8 C^{1 / 3}\left(\frac{M_{\mathrm{p}} / M_{\mathrm{s}}}{10^{-3}}\right)^{1 / 3}\left\{\frac{j^{11}}{(j-1)^{14}}\right\}^{1 / 18} \mathrm{AU} .
\end{aligned}
$$

Figure 12 shows exoplanets identified so far and the predicted location of the SSP in the $a_{\mathrm{p}}-M_{\mathrm{p}}$ plane for $C=10$. The SSP locates from $\sim 1 \mathrm{AU}$ to $\sim 30 \mathrm{AU}$ and shifts outward with increasing planetary mass. The red and black symbols show exoplanets observed using the transit method and the radial velocity method, respectively (http://exoplanet.eu). Given that planetary growth occurs via the runaway gas accretion, followed by inward migration, the evolution path of a gas giant planet can be drawn from right-bottom to left-top direction in the $a_{\mathrm{p}}-M_{\mathrm{p}}$ plane (e.g., Ida \& Lin 2004; Mordasini et al. 2009). If runaway gas accretion

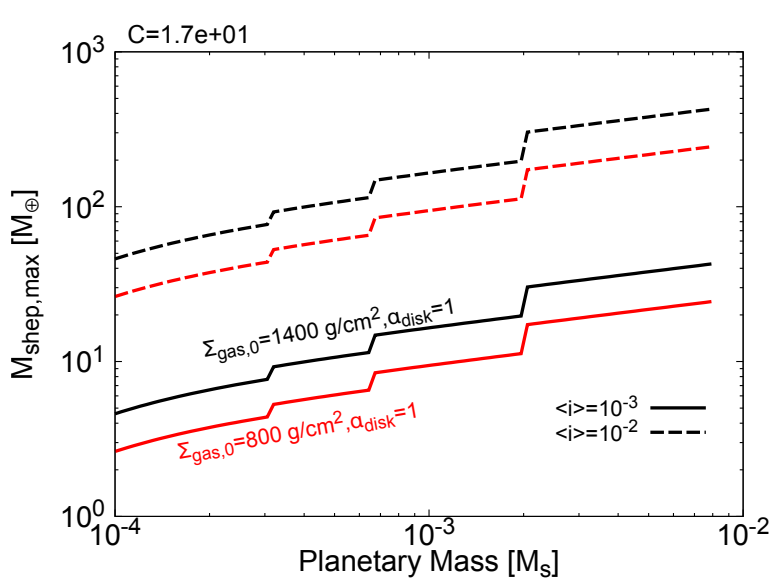

Fig. 13. The maximum mass of planetesimals shepherded into the SSP without active planetesimal collisions $M_{\text {max,shep }}$ as a function of planetary mass $M_{\mathrm{p}}$. Here, we consider the case where the protoplanet migrates with the type II regime obtained by Kanagawa et al. (2018) (see eq. (51). The solid and dashed lines show the cases of $\langle i\rangle=10^{-3}$ and $10^{-2}$, respectively. The black and red lines are cases of massive disk with $\Sigma_{\text {gas }, 0}=1400 \mathrm{~g} / \mathrm{cm}^{2}$ and $800 \mathrm{~g} / \mathrm{cm}^{2}$, respectively. We adapt the optically thick disk of $T_{0}=140 \mathrm{~K}$, the disk viscosity $\alpha_{\text {vis }}=10^{-3}$, the size of planetesimal $R_{\mathrm{pl}}=10^{7} \mathrm{~cm}$ and the planetesimal density $\rho_{\mathrm{pl}}=1 \mathrm{~g} / \mathrm{cm}^{3}$. The scaling parameter of the location of the SSP $C$ is obtained as $C=17$.

begins in outer disk region, which might be favoured for planetary core formation because of the increasing isolation mass of protoplanet (e.g. Armitage 2010), the exoplanets currently observed in the region interior to the SSP must have crossed the SSP during their formation stages.

\subsubsection{Maximum mass of planetesimals shepherded into the SSP}

The total mass of accreted planetesimals depends on the amount of planetesimals that shepherded into the SSP. By substituting eqs. (4), (54) and (62) into eq. (45), we can estimate $M_{\text {shep,max }}$ when the protoplanet reaches the outer edge of the SSP. In this case, $M_{\text {shep,max }}$ weakly depends on $\alpha_{\text {vis }}, \rho_{\mathrm{pl}}, R_{\mathrm{pl}}$ and $M_{\mathrm{p}}{ }^{-1}$ $(-1 / 4$ powers of each value), but linearly depends on $\tan \langle i\rangle$, $\Sigma_{\text {gas }, 0}$ and $T_{0}^{-1}$. Here, we adapt the optically thick disk of $T_{0}=140 \mathrm{~K}$ (Sasselov \& Lecar 2000) and consider two disks with $\Sigma_{\text {gas }, 0}=1400 \mathrm{~g} / \mathrm{cm}^{2}$ and $800 \mathrm{~g} / \mathrm{cm}^{2}$. The former disk corresponds to the massive disk of total mass $0.1 M_{\mathrm{s}}$ and typical radius $100 \mathrm{AU}$. Figure 13 shows $M_{\text {shep,max }}$ as a function of planetary mass $M_{\mathrm{p}} / M_{\mathrm{s}}$. We find that a few tens $M_{\oplus}$ of planetesimals can be shepherded into the SSP before initiating collisional cascade even with $\tan \langle i\rangle=10^{-3}$. For the capture of several tens or more planetesimals, the effect enhancing $\tan \langle i\rangle$ needs to be considered.

Note that $M_{\text {shep,max }}$ only provides the maximum mass of planetesimals shepherded into the SSP and not the mass of planetesimals that can be captured by the protoplanet. The numerical simulations we presented in sec. 3 suggest that $\sim 30 \%$ of the shepherded planetesimals are captured by the protoplanet. This accretion efficiency, however, depends on various parameters such as the inclination of planetesimals (Tanaka \& Ida 1999) and the planetary capture radius (Inaba \& Ikoma 2003). In addition, the initial semi-major axis of planetesimals also affects the capture efficiency because of the mean motion resonances. 
A detailed investigation of the accretion efficiency under various conditions should be conducted in future research.

\subsection{Heavy-element enrichment of giant planets}

If giant planets cross the sweet spot during the migration phase, planetesimal accretion could take place. If tens Earth-masses of planetesimals were shepherded into the SSP as shown in fig. 13 , we would expect a clear difference in the total heavy-element mass of planets depending on whether they migrated interior or exterior to the SSP. If such a difference between planetary populations is observed, it could confirm that planetesimal accretion is a significant process that takes place during planetary migration. However, once the collisional cascade occurs, the location of the SSP moves to the outer disk region and the metallicity difference between the populations would be reduced. It is therefore still unclear whether such a difference is detectable.

Currently, there are no estimated metallicities for giant exoplanets within the outer disk region (i.e., at distances $\gtrsim 10 \mathrm{AU}$ ). To estimate the metallicity of giant exoplanets, measurements of the radius, mass and age of the planet are required. At the moment, data of the masses and radii of cold giant planets are still unavailable. Nevertheless, the upcoming decades are expected to provide vast observations thanks to various missions. Wide field observations, such as TESS and Plato, will increase the number of cold giant planets (Ricker et al. 2014, Rauer et al. 2014). Follow-up observations using precise radial velocity measurement, such as HARPS (Mayor et al.2003) and ESPRESSO (Pepe et al. 2010), and precise transit measurement, such as NGTS (Wheatley et al. 2018) and WASP (Pollacco et al.2006), are suitable for obtaining the masses and radii of giant planets at large radial distances. In addition, high-dispersion coronagraphy technique (e.g. Kotani et al. 2020) could constrain the atmospheric composition of cold giant planets. Next generation observations will constrain the main source of heavy elements.

In this study, we focus on planetesimal accretion and consider it as the main source of heavy-element enrichment in gas giant planets. However, other various processes could also lead to heavy-element enrichment. The metallicity of the gaseous disk can be enriched by the sublimation of dust grains and pebbles crossing each ice lines (Booth et al. 2017; Booth \& Ilee 2019). The accretion of these enriched disk gas brings massive heavy elements into gas giant planets (Schneider \& Bitsch 2021). Giant impacts have also been investigated as a source of enrichment (Ikoma et al. 2006, Liu et al. 2015, 2019, Ginzburg \& Chiang 2020: Ogihara et al. 2021). By combining the effects of pebble accretion and giant impacts, Ogihara et al. (2021) found that up to $\sim 100 M_{\oplus}$ of heavy elements can be accreted by a giant planet through multi-giant impacts of Neptune-mass planets.

In order to determine what is the main source of the heavyelement enrichment in giant exoplanets, other observational information such as elemental ratios (refractory-to-volatile, $\mathrm{C} / \mathrm{O}$, $\mathrm{O} / \mathrm{H}$, etc) is valuable. In recent observations, the existences of $\mathrm{TiO}$ and VO in Hot-Jupiters is suggested by the transmission spectrum analysis (Evans et al. 2016; Chen et al. 2021). The amount of these refractory materials is useful for constraining the dominant process of heavy-element accretion. The accretion of massive refractory materials is only possible through solid accretion, thus using the information of refractory materials, we can constrain whether the heavy elements are brought by solid accretion or gas accretion.

The elemental ratio of volatile materials also provides information on the accretion process of heavy-elements (e.g., Madhusudhan et al. 2014, Notsu et al. 2020, Turrini et al. 2021;
Schneider \& Bitsch 2021). Turrini et al. (2021) investigated the effect of planetesimal accretion on elemental ratios of volatile materials such as $\mathrm{C} / \mathrm{O}, \mathrm{N} / \mathrm{O}$ or $\mathrm{S} / \mathrm{N}$. They considered a Jupitermass planet in a typical disk model, however, the relative position between the SSP and each ice lines changes with the planetary mass and disk conditions. The elemental ratio of planetesimals shepherded into the SSP would depend on the evolution pathways of the giant planets on the $a_{\mathrm{p}}-M_{\mathrm{p}}$ plane and disk conditions. We hope to further investigate the link between elemental ratios and the formation history in followup studies. This is highly relevant since future missions like JWST and Ariel will provide accurate measurements of the atmospheric composition of gaseous planets that can then be used to better understand the planetary origin.

\section{Summary \& Conclusions}

Planetesimal accretion during planetary migration could lead to a significant enrichment of giant planets with heavy elements. As revealed in Shibata et al. (2020), planetesimal accretion occurs in a sweet spot for planetesimal accretion (SSP), where planetesimals can be captured by the migrating planet efficiently. By performing dynamical simulations for planetesimals with a migrating planet, Shibata et al. (2020) demonstrated that the SSP emerges due to shepherding processes caused by aerodynamic gas drag and mean motion resonances. However, they conducted no detailed analysis of the nature (especially, the location) of the SSP and therefore in this paper we have focused on the nature of the accretion sweet spot.

In sec. 2. we analytically derived an equation describing the location of the SSPand found the following: (i) The SSP is regulated by the ratio of the damping timescale for the planetesimal eccentricity due to aerodynamic gas drag to the migration timescale of the planet (ii) The SSP also depends on the planetary mass, because the accretion bands change with the relative positions between the mean motion resonances and the feeding zone.

In sec. 3. we performed numerical simulations and confirmed that the derived conditions reproduce the numerical results well. In sec. 4, we discussed the effect of planetesimal collisions and ablation during the shepherding process. These effects would be important for inner disk region as $\lesssim 10 \mathrm{AU}$ and put other constraint for planetesimal accretion process. If the migration timescale is inversely proportional to the disk gas surface density (e.g., Kanagawa et al. 2018), the timescale ratio is rather insensitive to the structure of the protoplanetary disk. Therefore, the location of the SSP is fixed during the evolution of the protoplanetary disk.

Finally, we discussed the effect of the SSP on the heavyelement enrichment of gas giant planets. We suggest that the existence of the SSP makes a difference in the heavy-element mass between planets observed in the regions interior and exterior to the SSP. The detailed chemical composition of gas giant planets, such as refractory-to-volatile ratio or $\mathrm{C} / \mathrm{O}$ ratio, would be also affected by the location of the SSP. If such compositional features can be observed, it could be a piece of the evidence of planetesimal accretion during planetary migration. Future observations in the upcoming decades will reveal whether planetesimal accretion is a main source of heavy elements in giant planets, and therefore advance our understanding of their origin.

Acknowledgements. S. S. and R. H. acknowledge support from the Swiss National Science Foundation (SNSF) under grant 200020_188460. Part of this work was supported by the German Deutsche Forschungsgemeinschaft, DFG project number Ts $17 / 2-1$ and by JSPS Core-to-Core Program "International 
S. Shibata et al.: The origin of the high metallicity of close-in giant exoplanets II

Network of Planetary Sciences $\left(\right.$ Planet $^{2}$ )" and JSPS KAKENHI Grant Numbers $17 \mathrm{H} 01153$ and 18H05439. 


\section{Appendix A: Model descriptions}

\section{Appendix A.1: Equations of motion}

The equation of motion is given by:

$\frac{\mathrm{d} \mathbf{r}_{i}}{\mathrm{~d} t}=\sum_{i \neq j} \mathbf{f}_{\text {grav }, i, j}+\mathbf{f}_{\text {aero }}+\mathbf{f}_{\text {tide }}$,

where $\mathbf{r}_{i}$ is the position vector relative to the initial (i.e., $t=0$ ) mass centre of the star-planet-planetesimals system, $\mathbf{f}_{\text {grav }, i, j}$ is the mutual gravity between particles $i$ and $j$ given by:

$\mathbf{f}_{\mathrm{grav}, i, j}=-\mathcal{G} \frac{M_{j}}{r_{i, j}{ }^{3}} \mathbf{r}_{i, j}$

with $\mathbf{r}_{i, j}$ being the position vector of particle $i$ relative to particle $j\left(r_{i, j} \equiv\left|\mathbf{r}_{i, j}\right|\right), M_{j}$ is the mass of particle $j$, and $\mathcal{G}$ is the gravitational constant. $\mathbf{f}_{\text {aero }}$ is the aerodynamic gas drag, and $\mathbf{f}_{\text {tide }}$ is the gravitational tidal drag from the protoplanetary disk gas. The central star, planet, and planetesimals are denoted by the subscripts $i$ (or $j$ ) $=1,2$, and $\geq 3$, respectively. The planetesimals are treated as test particles; therefore $f_{\text {grav }, i, j}=0$ in Eq. A.1 for $j \geq 3$. The aerodynamic gas drag force and the tidal drag force are given respectively by:

$\mathbf{f}_{\text {aero }}=-\frac{\mathbf{u}}{\tau_{\text {aero }},}$
$\mathbf{f}_{\text {tide }}=-\frac{\mathbf{v}_{\mathrm{p}}}{2 \tau_{\text {tide }, a}}$,

with

$\tau_{\text {aero }}=\frac{2 m_{\mathrm{pl}}}{C_{\mathrm{d}} \pi R_{\mathrm{pl}}^{2} \rho_{\mathrm{gas}} u}=\tau_{\text {aero }, 0} \frac{v_{\mathrm{K}}}{u}$

Here $\mathbf{u}=\mathbf{v}_{\mathrm{pl}}-\mathbf{v}_{\text {gas }}(u=|\mathbf{u}|)$ is the planetesimal's velocity $\left(\mathbf{v}_{\mathrm{pl}}\right)$ relative to the ambient gas $\left(\mathbf{v}_{\text {gas }}\right)$, Given the range of the planetesimal mass $\left(\sim 10^{16}-10^{22} \mathrm{~g}\right)$ and planet $\left(\sim 10^{30} \mathrm{~g}\right)$, we assume $f_{\text {tide }}=0$ for the former and $f_{\text {aero }}=0$ for the latter. The central star is not affected by $f_{\text {aero }}$ and $f_{\text {tide }}$.

\section{Appendix A.2: Gas disk}

The protoplanetary disk is assumed to be vertically isothermal, and the gas density $\rho_{\text {gas }}$ is expressed as:

$\rho_{\text {gas }}=\frac{\Sigma_{\text {gas }}}{\sqrt{2 \pi} h_{\mathrm{s}}} \exp \left(-\frac{z^{2}}{2 h_{\mathrm{s}}^{2}}\right)$,

where $z$ is the height from the disk mid-plane and $h_{\mathrm{s}}$ is the disk's scale height. The aspect ratio of the protoplanetary disk is given by:

$\frac{h_{\mathrm{s}}}{r}=\frac{c_{\mathrm{s}}}{r \Omega_{\mathrm{K}}}$

where $\Omega_{\mathrm{K}}$ is the Kepler angular velocity and $c_{\mathrm{S}}$ is the isothermal sound speed of disk gas. The sound speed is given as

$c_{\mathrm{s}}=\sqrt{\frac{k_{\mathrm{B}} T_{\mathrm{disk}}}{\mu_{\mathrm{disk}} m_{\mathrm{H}}}} \propto r^{-\beta \mathrm{disk}}$,

where $k_{\mathrm{B}}$ is the Boltzmann constant and $\mu_{\text {disk }}$ is the mean molecular weight in the unit of proton mass $m_{\mathrm{H}}$. In this study, $\mu_{\text {disk }}$ is set as 2.3 and the aspect ratio of the disk gas at $1 \mathrm{AU}$ is $\sim 0.03$.
The gas in the protoplanetary disk rotates with a subKeplerian velocity because of pressure gradient; namely, $v_{\text {gas }}=$ $\left(1-\eta_{\text {gas }}\right) r \Omega_{\mathrm{K}}$, where $\eta_{\text {gas }}$ is $\ll 1$ and given as

$$
\begin{aligned}
\eta_{\text {gas }} & \equiv-\frac{1}{2}\left(\frac{h_{\mathrm{s}}}{r}\right)^{2} \frac{\mathrm{d} \ln P_{\mathrm{gas}}}{\mathrm{d} \ln r} \\
& =\frac{1}{2}\left(\frac{h_{\mathrm{s}}}{r}\right)^{2}\left[\frac{3}{2}\left(1-\frac{z^{2}}{h_{\mathrm{s}}^{2}}\right)+\alpha_{\text {disk }}+\beta_{\text {disk }}\left(1+\frac{z^{2}}{h_{\mathrm{s}}^{2}}\right)\right]
\end{aligned}
$$

$P_{\text {gas }}$ is the gas pressure.

\section{Appendix A.3: Planetesimals}

The surface number density of planetesimals is given as $\Sigma_{\text {solid }} / m_{\mathrm{pl}}$ which gives $\sim 10^{6} / \mathrm{AU}^{2}$ at maximum. To speed up the numerical integration, we follow the orbital motion of superparticles. The surface number density of super-particles $n_{\mathrm{s}}$ is given as:

$n_{\mathrm{s}}=n_{\mathrm{s}, 0}\left(\frac{r}{1 \mathrm{AU}}\right)^{-\alpha_{\mathrm{sp}}}$

where

$n_{\mathrm{s}, 0}=\frac{N_{\mathrm{sp}}}{2 \pi} \frac{2-\alpha_{\mathrm{sp}}}{\left(a_{\mathrm{pl}, \mathrm{out}} / 1 \mathrm{AU}\right)^{2-\alpha_{\mathrm{sp}}}-\left(a_{\mathrm{pl}, \text { in }} / 1 \mathrm{AU}\right)^{2-\alpha_{\mathrm{sp}}}}\left[\frac{1}{\mathrm{AU}^{2}}\right] ;$

$N_{\text {sp }}$ is the total number of super-particles used in a given simulation. In our simulation, we set $N_{\mathrm{sp}}=12,000$ and $\alpha_{\mathrm{sp}}=1$ to distribute super-particles uniformly in the radial direction. The mass per super-particle $M_{\mathrm{sp}}$ is given by:

$M_{\mathrm{sp}}\left(a_{0}\right)=\frac{\Sigma_{\text {solid }}\left(a_{0}\right)}{n_{\mathrm{s}}\left(a_{0}\right)}$,

where $a_{0}$ is the initial semi-major axis of the super-particle.

As for eccentricity and inclination, although planetesimals are treated as test particles in our simulations, assuming that planetesimals are, in reality, scattered by their mutual gravitational interaction, we adopt the Rayleigh distribution as the initial eccentricities $e$ and inclinations $i$ of planetesimals. We set $\left\langle e^{2}\right\rangle^{1 / 2}=2\left\langle i^{2}\right\rangle^{1 / 2}=10^{-3}$. The orbital angles $\Omega, \varpi$ and $\epsilon$ are distributed uniformly.

During the orbital integration, we judge that a super-particle has been captured by the planet once (i) the super-particle enters the planet's envelope or (ii) its Jacobi energy becomes negative in the Hill sphere. The planet's radius $R_{\mathrm{p}}$ is calculated as:

$R_{\mathrm{p}}=\left(\frac{3 M_{\mathrm{p}}}{4 \pi \rho_{\mathrm{p}}}\right)^{1 / 3}$,

where $\rho_{\mathrm{p}}$ is the planet's mean density. The planet's radius is assumed to be extended shortly after formation and the density is set to $\rho_{\mathrm{p}}=0.125 \mathrm{~g} \mathrm{~cm}^{-3}$, corresponding to a planet's radius twice as large as Jupiter's current radius.

\section{References}

Aarseth, S. J. 1985, in IAU Symposium, Vol. 113, Dynamics of Star Clusters, ed. J. Goodman \& P. Hut, 251-258

Adachi, I., Hayashi, C., \& Nakazawa, K. 1976, Progress of Theoretical Physics, 56,1756

Alibert, Y., Mordasini, C., Benz, W., \& Winisdoerffer, C. 2005, A\&A, 434, 343 Armitage, P. J. 2010, Astrophysics of Planet Formation 
Batygin, K. 2015, Monthly Notices of the Royal Astronomical Society, 451, 2589

Batygin, K. \& Laughlin, G. 2015, Proceedings of the National Academy of Sciences of the United States of America, 112, 4214

Benz, W. \& Erik, A. 1999, Icarus, 142, 5

Booth, R. A., Clarke, C. J., Madhusudhan, N., \& Ilee, J. D. 2017, Monthly Notices of the Royal Astronomical Society, 469, 3994

Booth, R. A. \& Ilee, J. D. 2019, Monthly Notices of the Royal Astronomical Society, 487, 3998

Chen, G., Pallé, E., Parviainen, H., Murgas, F., \& Yan, F. 2021, The Astrophysical Journal Letters, 913, L16

D’Angelo, G. \& Podolak, M. 2015, ApJ, 806, 203

Debras, F. \& Chabrier, G. 2019, The Astrophysical Journal, 872, 100

Dermott, S. F., Malhotra, R., \& Murray, C. D. 1988, icarus, 76, 295

Dohnanyi, J. S. 1969, J. Geophys. Res., 74, 2531

Eriksson, L. E. J., Ronnet, T., \& Johansen, A. 2021, Astron. Astrophys. Suppl. Ser., 648, A112

Evans, T. M., Sing, D. K., Wakeford, H. R., et al. 2016

Fabrycky, D. C., Lissauer, J. J., Ragozzine, D., et al. 2014, Astrophysical Journal, 790, 146

Ginzburg, S. \& Chiang, E. 2020, Monthly Notices of the Royal Astronomical Society, 498, 680

Goldreich, P. 1965, Monthly Notices of the Royal Astronomical Society, 130, 159

Goldreich, P. \& Schlichting, H. E. 2014, The Astronomical Journal, 147, 32

Guillot, T. 1999, Planetary and Space Science, 47, 1183

Guillot, T., Santos, N. C., Pont, F., et al. 2006, Astronomy \& Astrophysics, 453, L21

Guo, K. \& Kokubo, E. 2021 [arXiv:2106.06240]

Hayashi, C. 1981, Progress of Theoretical Physics Supplement, 70, 35

Hayashi, C., Nakazawa, K., \& Adachi, I. 1977, Publication of Astronomical Society of Japan, 29, 163

Helled, R. \& Guillot, T. 2013, ApJ, 767, 113

Helled, R., Werner, S., Dorn, C., et al. 2021, Experimental Astronomy [arXiv:2103.08481]

Ida, S. \& Lin, D. N. C. 2004, The Astrophysical Journal, 604, 388

Ida, S. \& Nakazawa, K. 1989, Astronomy \& Astrophysics, 220, 293

Ida, S., Tanaka, H., Johansen, A., Kanagawa, K. D., \& Tanigawa, T. 2018, The Astrophysical Journal, 864, 77

Ikoma, M., Guillot, T., Genda, H., Tanigawa, T., \& Ida, S. 2006, The Astrophysical Journal, 650, 1150

Inaba, S. \& Ikoma, M. 2003, Astronomy \& Astrophysics, 410, 711

Kanagawa, K. D., Muto, T., Tanaka, H., et al. 2016, Publications of the Astronomical Society of Japan, 68, 43

Kanagawa, K. D., Tanaka, H., Muto, T., \& Tanigawa, T. 2017, Publications of the Astronomical Society of Japan, 69

Kanagawa, K. D., Tanaka, H., Muto, T., Tanigawa, T., \& Takeuchi, T. 2015, Monthly Notices of the Royal Astronomical Society, 448, 994

Kanagawa, K. D., Tanaka, H., \& Szuszkiewicz, E. 2018, The Astrophysical Journal, 861,140

Kotani, T., Kawahara, H., Ishizuka, M., et al. 2020, 11448, 1144878

Leinhardt, Z. M. \& Stewart, S. T. 2009, Icarus, 199, 542

Lin, D. N. C. \& Papaloizou, J. C. B. 1993, in Protostars and Planets III, ed. E. H. Levy \& J. I. Lunine, 749-835

Liu, S. F., Agnor, C. B., Lin, D. N., \& Li, S. L. 2015, Monthly Notices of the Royal Astronomical Society, 446, 1685

Liu, S.-F., Hori, Y., Müller, S., et al. 2019, Nature, 572, 355

Lynden-Bell, D. \& Pringle, J. E. 1974, MNRAS, 168, 603

Madhusudhan, N., Amin, M. A., \& Kennedy, G. M. 2014, The Astrophysical Journal Letters, 794, 12

Makino, J. \& Aarseth, S. J. 1992, Publications of the Astronomical Society of Japan, 44, 141

Malhotra, R. 1993a, Icarus, 106, 264

Malhotra, R. 1993b, Nature, 365, 819

Mankovich, C. \& Fuller, J. 2021 [arXiv: 2104.13385]

Mayor, M., Pepe, F., Queloz, D., et al. 2003, The Messenger, 114, 20

Miller, N. \& Fortney, J. J. 2011, The Astrophysical Journal, 736, L29

Mordasini, C. 2018, in Handbook of Exoplanets, 2425-2474

Mordasini, C., Alibert, Y., \& Benz, W. 2009, Astronomy \& Astrophysics, 501, 1139

Movshovitz, N., Fortney, J. J., Mankovich, C., Thorngren, D., \& Helled, R. 2020, Astrophys. J., 891, 109

Murray, C. D. \& Dermott, S. F. 1999, Solar system dynamics (Cambridge, UK: Cambridge University Press)

Nagasawa, M., Tanaka, K. K., Tanaka, H., et al. 2019, ApJ, 871, 110

Notsu, S., Eistrup, C., Walsh, C., \& Nomura, H. 2020, Monthly Notices of the Royal Astronomical Society, 499, 2229

Ogihara, M., Hori, Y., Kunitomo, M., \& Kurosaki, K. 2021, Astronomy \& Astrophysics, 648, L1

Ohtsuki, K., Stewart, G. R., \& Ida, S. 2002, Icarus, 155, 436
Pepe, F. A., Cristiani, S., Rebolo Lopez, R., et al. 2010, in Society of PhotoOptical Instrumentation Engineers (SPIE) Conference Series, Vol. 7735, Ground-based and Airborne Instrumentation for Astronomy III, ed. I. S. McLean, S. K. Ramsay, \& H. Takami, 77350F

Podolak, M., Haghighipour, N., Bodenheimer, P., Helled, R., \& Podolak, E. 2020, The Astrophysical Journal, 899, 45

Pollacco, D., Skillen, I., Cameron, A., et al. 2006, 304, 253

Pollack, J. B., Podolak, M., Bodenheimer, P., \& Christofferson, B. 1986, Icarus, 67, 409

Rauer, H., Catala, C., Aerts, C., et al. 2014, Exp. Astron., 38, 249

Ricker, G. R., Winn, J. N., Vanderspek, R., et al. 2014, in Society of PhotoOptical Instrumentation Engineers (SPIE) Conference Series, Vol. 9143, Space Telescopes and Instrumentation 2014: Optical, Infrared, and Millimeter Wave, ed. J. Oschmann, Jacobus M., M. Clampin, G. G. Fazio, \& H. A. MacEwen, 914320

Sasselov, D. D. \& Lecar, M. 2000, ApJ, 528, 995

Saumon, D. \& Guillot, T. 2004, The Astrophysical Journal, 609, 1170

Schneider, A. D. \& Bitsch, B. 2021, How drifting and evaporating pebbles shape giant planets I: Heavy element content and atmospheric C/O, Tech. rep.

Shakura, N. I. \& Sunyaev, R. A. 1973, Astronomy \& Astrophysics, 24, 337

Shibata, S., Helled, R., \& Ikoma, M. 2020, A\&A, 633, 13

Shibata, S. \& Ikoma, M. 2019, MNRAS, 487, 4510

Shiraishi, M. \& Ida, S. 2008, The Astrophysical Journal, 684, 1416

Tanaka, H. \& Ida, S. 1997, Icarus, 125, 302

Tanaka, H. \& Ida, S. 1999, Icarus, 139, 350

Tanaka, K. K., Yamamoto, T., Tanaka, H., et al. 2013, ApJ, 764, 120

Thorngren, D. P., Fortney, J. J., Murray-Clay, R. A., \& Lopez, E. D. 2016, The Astrophysical Journal, 831, 64

Turrini, D., Schisano, E., Fonte, S., et al. 2021, Astrophys. J., 909, 40

Wahl, S. M., Hubbard, W. B., Militzer, B., et al. 2017, Geophys. Res. Lett., 44, 4649

Wheatley, P. J., West, R. G., Goad, M. R., et al. 2018, Monthly Notices of the Royal Astronomical Society, 475, 4476

Yu, Q. \& Tremaine, S. 2001, The Astronomical Journal, 121, 1736

Zhou, J. \& Lin, D. N. C. 2007, The Astrophysical Journal, 666, 447 